\title{
Nonlinear convex and concave relaxations for the solutions of parametric ODEs
}

\author{
Joseph. K. Scott ${ }^{1}$, Benoit Chachuat ${ }^{2}$ and Paul. I. Barton ${ }^{1 *}$ \\ ${ }^{1}$ Process Systems Engineering Laboratory, Department of Chemical Engineering, Massachusetts Institute of \\ Technology, Cambridge, MA 02139, USA ${ }^{2}$ Centre for Process Systems Engineering, Department of Chemical \\ Engineering, Imperial College, London, SW7 2AZ, UK
}

\begin{abstract}
SUMMARY
Convex and concave relaxations for the parametric solutions of ordinary differential equations (ODEs) are central to deterministic global optimization methods for nonconvex dynamic optimization and open-loop optimal control problems with control parametrization. Given a general system of ODEs with parameter dependence in the initial conditions and right-hand sides, this work derives sufficient conditions under which an auxiliary system of ODEs describes convex and concave relaxations of the parametric solutions, pointwise in the independent variable. Convergence results for these relaxations are also established. A fully automatable procedure for constructing an appropriate auxiliary system has been developed previously by the authors. Thus, the developments here lead to an efficient, automatic method for computing convex and concave relaxations for the parametric solutions of a very general class nonlinear ODEs. The proposed method is presented in detail for a simple example problem. Copyright (c) 0000 John Wiley \& Sons, Ltd.
\end{abstract}

Received ...

KEY WORDS: Dynamic optimization; Optimal control; Global optimization; Ordinary differential equations

\section{INTRODUCTION}

The purpose of this work is to present a theoretical result which enables convex and concave relaxations of the solutions of parametric ordinary differential equations (ODEs) to be constructed. In particular, a general system of ODEs is considered where both the initial conditions and the righthand side functions depend on a real parameter vector. Given such a system, an auxiliary system of ODEs is derived which describes convex underestimators and concave overestimators for each of the state variables with respect to the parameters, pointwise in the independent variable.

The primary motivation for this construction is its application in algorithms for the deterministic global optimization of physical systems which are described by systems of ODEs, typically referred to as dynamic optimization or optimal control problems [1, 2, 3]. A standard approach

*Correspondence to: 66-464, 77 Massachusetts Ave., Cambridge, MA 02139, USA. E-mail:pib@mit.edu

Copyright (c) 0000 John Wiley \& Sons, Ltd.

Prepared using ocaauth.cls [Version: 2010/03/27 v2.00] 
to computational optimal control is to apply control parametrization, which replaces the control functions by an approximate representation in terms of a finite number of real parameters, such as piecewise constant or piecewise linear controls [4, 5]. Time optimal control problems can also be transformed into fixed time problems through the introduction of a real scaling parameter [6]. These reformulations replace the original control system with a nonlinear system of parametric ODEs of the type considered in this article. Furthermore, in the case where the solution of these ODEs is unique for any fixed parameter vector and initial condition, this reformulation yields a Euclidean optimization problem, i.e., an optimization problem in which the feasible set is a subset of a Euclidean space as opposed to a function space. Thus, control parametrization yields an approximation to the original optimal control problem which is amenable to computational methods and practical implementation because it can be represented by finite data. For a detailed discussion of the convergence properties of such approximations and a precise description of the wide class of optimal control problems for which this methodology can be applied, the reader is referred to [4].

As is the case with more standard Euclidean optimization problems, the global solution of an optimal control problem reformulated through control parametrization can be obtained by solving a sequence of convex underestimating programs within a branch-and-bound algorithm [7, 8]. Convex underestimating programs are also used in global optimization algorithms for mixed-integer nonlinear programs based on outer approximation techniques [9], and the extension to mixed-integer dynamic optimization problems has been developed [10]. However, the presence of embedded differential equations precludes the use of standard techniques $[8,11,12]$ for generating these convex underestimating programs. The primary complication is exactly the task addressed in this work; the generation of convex underestimators and concave overestimators for the solutions of the ODEs themselves.

In recent years, a few authors have proposed methods for generating convex and concave relaxations for the solutions of ODEs. The first method was proposed by Esposito and Floudas [13] using a dynamic extension of the $\alpha B B$ convexification theory described in [12]. This method relies on a finite sampling step to bound the second-order sensitivities of the ODEs, and therefore cannot guarantee that the resulting relaxations are convex. In [3], bounds on these sensitivities are computed, resulting in guaranteed convex relaxations, yet these relaxations are typically very weak and the second-order sensitivities are costly to evaluate. Singer and Barton [14] presented a theory for generating overestimators and underestimators for the solutions of ODEs which, by construction, are affine in the parameters, and so are trivially convex and concave. These affine relaxations are computed as the solutions of a auxiliary system of linear time-varying ODEs which can be automatically constructed using McCormick's relaxation technique and outer-linearization [15]. Because these auxiliary ODEs can be solved using standard numerical integration codes, these relaxations can be evaluated relatively efficiently; i.e., at a cost comparable to integration of the original ODE model. Moreover, these affine relaxations prove to be much stronger than the relaxations described in [3]. However, affine relaxations are often unsatisfactory for underestimating (overestimating) ODE solutions which are highly nonlinear in the parameters. Furthermore, constructing these relaxations requires the specification of a reference trajectory which typically has a large impact on the quality of the resulting relaxations. More recently, two related approaches have been developed in which McCormick's relaxation technique is applied to a characterization of the ODE solution by a Taylor expansion with a rigorous enclosure of the truncation error $[16,17]$. 
These methods extend interval bounding techniques based on a similar analysis [18] and appear capable of providing very tight relaxations when a sufficiently high-order expansion is used. On the other hand, computing relaxations of a high-order Taylor expansion is very expensive for high dimensional problems, and the existence an appropriate compromise in the context of global optimization remains an open question. On the whole, global dynamic optimization solvers can presently only solve relatively small problems in reasonable computational time due to the lack of an efficient method for computing tight convex and concave relaxations [2, 3, 13, 18].

In this article, we present a method for computing nonlinear convex and concave relaxations through the solution of an auxiliary system of nonlinear ODEs. The initial conditions and righthand side functions of this auxiliary system are constructed automatically by an application of the generalized McCormick relaxation technique developed by the authors [19]. Like the affine relaxation theory in [14], evaluating these relaxations involves a single numerical integration, so the cost is comparable to a single integration of the original ODEs. However, the resulting relaxations are potentially non-affine, and hence provide better relaxations for highly nonlinear ODE solutions. Moreover, they do not require a priori knowledge of an appropriate reference trajectory.

Several other seemingly related notions of convexity and relaxation appear in the literature on optimal control and ODE theory which are relevant to this work in varying degrees. In [20], sufficient conditions are given under which an optimal control problem on a general Hilbert space is convex, based on classical results on the composition of convex functions. If this Hilbert space is taken as a finite-dimensional real vector space, as would result from reformulation through control parametrization [4], this notion of convexity is equivalent to that in the work presented here. However, the conditions in [20] are extremely restrictive, and no constructive procedure is given for generating convex and concave relaxations of nonconvex problems. In more classical results regarding sufficient optimality conditions for optimal control problems [1,21], convexity of the Hamiltonian is assumed with respect to the state variables and the controls. Convexity in this sense treats the states and controls as unrelated, whereas the purpose of this work is to approximate the parametric dependence of the state variables by convex and concave functions, so these notions are distinct. The article [22] (and the references therein) details conditions for the reachable set of a system of ODEs beginning from a ball of initial conditions to be convex. Again, this is an unrelated notion because a convex set in state space does not imply convex dependence on the initial conditions for each state variable, nor the converse. Finally, the term relaxation is often applied to optimal control and variational problems where the set of admissible controls is enlarged or embedded in a larger space (i.e., measure-valued controls), and/or the cost functional is underestimated by a lower semicontinuous functional [23, 24]. Though similar in spirit, the type of relaxations considered here are fundamentally different (see Definition 2.1).

The remainder of this article is organized as follows. Section 2 contains preliminary definitions and results. In $\S 3$, the system of differential equations to be relaxed is defined and all necessary assumptions are stated. Section 4 contains the main contributions of the article. The main result is a set of sufficient conditions for an auxiliary system of differential equations to have solutions which are the desired convex and concave state relaxations. Under further assumptions on this auxiliary system, it is proven that the resulting relaxations may be used to construct lower bounding problems for branch-and-bound procedures applied to global dynamic optimization problems, and the resulting algorithm is finite $\varepsilon$-convergent. Section 5 discusses the automatic computation of 
an appropriate auxiliary system and other implementation issues. Finally, $\S 6$ demonstrates these relaxations for a simple example problem, and it is shown that they approximate the parametric solution well.

\section{PRELIMINARIES}

Throughout this article, vector quantities are denoted in bold, while scalar quantities are written without emphasis. For any $\mathbf{v} \in \mathbb{R}^{n}$, the standard $p$-norms are denoted by $\|\mathbf{v}\|_{p}=\left(\sum_{i=1}^{n}\left|v_{i}\right|^{p}\right)^{1 / p}$, $1 \leq p<\infty$, and $\|\mathbf{v}\|_{\infty}=\max _{i}\left|v_{i}\right|$. Suppose that $\mathbf{w}, \mathbf{u} \in \mathbb{R}^{n}$ as well. The order relations $\mathbf{v} \leq \mathbf{w}$ and $\mathbf{v}<\mathbf{w}$ denote that these relations hold elementwise. Similarly, $\min (\mathbf{v}, \mathbf{w})$ and $\max (\mathbf{v}, \mathbf{w})$ denote the vectors with elements $\min \left(v_{i}, w_{i}\right)$ and $\max \left(v_{i}, w_{i}\right)$, respectively, and $\operatorname{mid}(\mathbf{v}, \mathbf{w}, \mathbf{u})$ denotes the vector where each element is the middle value of $v_{i}, w_{i}$ and $u_{i}$. Finally, if a vector function is referred to as convex (concave), it is intended to mean that the scalar functions describing each element of the vector are convex (concave).

This work involves the construction of convex and concave relaxations, defined as follows.

Definition 2.1

Let $P$ be a convex set in $\mathbb{R}^{n_{p}}$ and $g: P \rightarrow \mathbb{R}$. A function $g^{c}: P \rightarrow \mathbb{R}$ is a convex relaxation, or convex underestimator, of $g$ on $P$ if $g^{c}$ is convex on $P$ and $g^{c}(\mathbf{p}) \leq g(\mathbf{p})$ for all $\mathbf{p} \in P$. Similarly, a function $g^{C}: P \rightarrow \mathbb{R}$ is a concave relaxation, or concave overestimator, of $g$ on $P$ if $g^{C}$ is concave on $P$ and $g^{C}(\mathbf{p}) \geq g(\mathbf{p})$ for all $\mathbf{p} \in P$.

The following theorem from [2] is a key result which enables the construction of nonlinear convex and concave relaxations for the solutions of parametric ODEs.

\section{Theorem 2.1}

Let $I=\left[t_{0}, t_{f}\right] \subset \mathbb{R}$, let $P \subset \mathbb{R}^{n_{p}}$ be convex, and let $\ell: I \times P \rightarrow \mathbb{R}$. If $\ell(\cdot, \mathbf{p})$ is Lebesgue integrable on $I$ for each $\mathbf{p} \in P$ and $\ell(t, \cdot)$ is convex on $P$ (resp. concave on $P$ ) for almost every $t \in I$, then the mapping

$$
P \ni \mathbf{p} \longmapsto L(\mathbf{p}) \equiv \int_{t_{0}}^{t_{f}} \ell(t, \mathbf{p}) d t
$$

is convex on $P$ (resp. concave on $P$ ).

\section{PROBLEM STATEMENT}

The following definition describes the general form of the ordinary differential equations which may be relaxed by the method described in this work. All necessary assumptions are subsequently stated.

Definition $3.1\left(I, P, D, \mathbf{f}, \mathbf{x}_{0}, \mathbf{x}\right)$

Let $I=\left[t_{0}, t_{f}\right] \subset \mathbb{R}, P \subset \mathbb{R}^{n_{p}}$ be a closed, bounded $n_{p}$-dimensional interval, and $D \subset \mathbb{R}^{n_{x}}$ be an open connected set. Further, consider the mappings $\mathbf{f}: I \times P \times D \rightarrow \mathbb{R}^{n_{x}}$ and $\mathbf{x}_{0}: P \rightarrow D$ and 
define the initial-value problem in parametric ordinary differential equations:

$$
\dot{\mathbf{x}}(t, \mathbf{p})=\mathbf{f}(t, \mathbf{p}, \mathbf{x}(t, \mathbf{p})), \quad \mathbf{x}\left(t_{0}, \mathbf{p}\right)=\mathbf{x}_{0}(\mathbf{p}),
$$

where a solution of (1) is any continuous mapping $\mathbf{x}: I \times P \rightarrow D$ such that, for any $\mathbf{p} \in P$, the mapping $\mathbf{x}(\cdot, \mathbf{p})$ is differentiable and satisfies (1) everywhere on $I$ (with derivatives from the right and left at $t_{0}$ and $t_{f}$, respectively).

Assumption 3.1

The ODEs (1) satisfy the following conditions:

1. $\mathrm{x}_{0}$ is continuous on $P$,

2. $\mathbf{f}$ is continuous on $I \times P \times D$,

3. for any compact $K \subset D, \exists L_{K} \in \mathbb{R}_{+}$such that

$$
\|\mathbf{f}(t, \mathbf{p}, \mathbf{z})-\mathbf{f}(t, \mathbf{p}, \hat{\mathbf{z}})\|_{1} \leq L_{K}\|\mathbf{z}-\hat{\mathbf{z}}\|_{1}, \quad \forall(t, \mathbf{p}, \mathbf{z}, \hat{\mathbf{z}}) \in I \times P \times K \times K .
$$

For any compact $K \subset D$, a function satisfying the inequality of Condition 3 in Assumption 3.1 is said to be Lipschitz on $K$ uniformly on $I \times P$.

\section{Remark 3.1}

The parameters $\mathbf{p}$ in (1) are assumed to take values in an $n_{p}$-dimensional closed, bounded interval. This is done primarily for computational reasons, though the theoretical developments to follow could deal just as easily with a more general compact, convex set in $\mathbb{R}^{n_{p}}$. In particular, McCormick's relaxation technique [11] requires that the parameter space be an interval.

Under Assumption 3.1, a standard proof demonstrates that, for small enough $d \in\left[0, t_{f}-t_{0}\right]$, there exists a unique solution of (1) on $\left[t_{0}, t_{0}+d\right] \times P$ (by simple parametric extension of, for example, Theorem 3.1 in Ch.1 of [25] or Theorem 3.1 in [26]). However, this result is only local. Naturally, we are only concerned with constructing relaxations of solutions where they exist and are unique. Therefore, we make the following assumption.

\section{Assumption 3.2}

A unique solution of (1), $\mathrm{x}$, exists on all of $I \times P$.

The objective of this work is to construct state relaxations for (1), defined as follows.

\section{Definition 3.2}

Two continuous functions $\mathbf{c}, \mathbf{C}: I \times P \rightarrow \mathbb{R}^{n_{x}}$ are state relaxations for (1) on $P$ if $\mathbf{c}(t, \cdot)$ and $\mathbf{C}(t, \cdot)$ are, respectively, convex and concave relaxations of $\mathbf{x}(t, \cdot)$ on $P$, for every fixed $t \in I$.

\section{THEORETICAL DEVELOPMENT}

In this section, sufficient conditions are given for the right-hand side functions and initial conditions of an auxiliary system of ODEs to have solutions which are state relaxations for (1) on $P$. Consider the following auxiliary ODEs. 
Definition $4.1\left(\mathbf{u}, \mathbf{o}, \mathbf{c}_{0}, \mathbf{C}_{0}\right)$

Let $\mathbf{c}_{0}, \mathbf{C}_{0}: P \rightarrow \mathbb{R}^{n_{x}}$ and $\mathbf{u}, \mathbf{o}: I \times P \times \mathbb{R}^{n_{x}} \times \mathbb{R}^{n_{x}} \rightarrow \mathbb{R}^{n_{x}}$, and define the auxiliary initial value problem in parametric ODEs:

$$
\begin{array}{rll}
\dot{\mathbf{c}}(t, \mathbf{p}) & =\mathbf{u}(t, \mathbf{p}, \mathbf{c}(t, \mathbf{p}), \mathbf{C}(t, \mathbf{p})), & \mathbf{c}\left(t_{0}, \mathbf{p}\right)=\mathbf{c}_{0}(\mathbf{p}), \\
\dot{\mathbf{C}}(t, \mathbf{p}) & =\mathbf{o}(t, \mathbf{p}, \mathbf{c}(t, \mathbf{p}), \mathbf{C}(t, \mathbf{p})), & \mathbf{C}\left(t_{0}, \mathbf{p}\right)=\mathbf{C}_{0}(\mathbf{p}),
\end{array}
$$

for all $(t, \mathbf{p}) \in I \times P$.

Assumption 4.1

The ODEs (2) satisfy the following conditions:

1. $\mathbf{c}_{0}$ and $\mathbf{C}_{0}$ are continuous on $P$,

2. $\mathbf{u}$ and $\mathbf{o}$ are continuous on $I \times P \times \mathbb{R}^{n_{x}} \times \mathbb{R}^{n_{x}}$,

3. $\exists L_{\text {uo }} \in \mathbb{R}_{+}$such that

$$
\begin{aligned}
\|\mathbf{u}(t, \mathbf{p}, \mathbf{z}, \mathbf{y})-\mathbf{u}(t, \mathbf{p}, \hat{\mathbf{z}}, \hat{\mathbf{y}})\|_{1}+\quad\|\mathbf{o}(t, \mathbf{p}, \mathbf{z}, \mathbf{y})-\mathbf{o}(t, \mathbf{p}, \hat{\mathbf{z}}, \hat{\mathbf{y}})\|_{1} \\
\leq L_{\mathbf{u o}}\left(\|\mathbf{z}-\hat{\mathbf{z}}\|_{1}+\|\mathbf{y}-\hat{\mathbf{y}}\|_{1}\right)
\end{aligned}
$$

for all $(t, \mathbf{p}, \mathbf{z}, \mathbf{y}, \hat{\mathbf{z}}, \hat{\mathbf{y}}) \in I \times P \times \mathbb{R}^{n_{x}} \times \mathbb{R}^{n_{x}} \times \mathbb{R}^{n_{x}} \times \mathbb{R}^{n_{x}}$.

The following definition gives sufficient conditions for the solutions of (2) to describe the desired convex and concave relaxations of $\mathrm{x}$ (see Theorem 4.1). Functions satisfying these conditions, as well as those of Assumption 4.1, can be constructed automatically using only knowledge of the functions $\mathbf{f}$ and $\mathbf{x}_{0}$. This construction is the subject of $\S 5$.

\section{Definition 4.2}

The auxiliary system of ODEs (2) is called a C-system of (1) on $P$ if, in addition to satisfying Assumption 4.1, the following conditions hold:

1. $\mathbf{c}_{0}$ and $\mathbf{C}_{0}$ are, respectively, convex and concave relaxations of $\mathbf{x}_{0}$ on $P$,

2. for any continuous mappings $\phi, \psi: I \times P \rightarrow \mathbb{R}^{n_{x}}$ and any fixed $t \in I$, the functions $\mathbf{u}(t, \cdot, \boldsymbol{\phi}(t, \cdot), \boldsymbol{\psi}(t, \cdot))$ and $\mathbf{o}(t, \cdot, \boldsymbol{\phi}(t, \cdot), \boldsymbol{\psi}(t, \cdot))$ are, respectively, convex and concave relaxations of $\mathbf{f}(t, \cdot, \mathbf{x}(t, \cdot))$ on $P$, provided that $\phi(t, \cdot)$ and $\psi(t, \cdot)$ are, respectively, convex and concave relaxations of $\mathbf{x}(t, \cdot)$ on $P$.

The following theorem is the central result of this work. It is shown that if (2) is a C-system of (1) on $P$, then the unique solution of (2) provides the desired state relaxations for (1) on all of $I \times P$. The proof uses a standard construction in ODE theory known as successive approximations (or Picard iterates) [25]. In particular, Theorem A.1 in Appendix A is required.

\section{Theorem 4.1}

If the auxiliary system of ODEs (2) is a C-system of (1) on $P$, then $\mathbf{c}(t, \cdot)$ and $\mathbf{C}(t, \cdot)$ are, respectively, convex and concave relaxations of $\mathbf{x}(t, \cdot)$ on $P$, for each fixed $t \in I$.

\section{Proof}

Choose any two vectors in $\mathbb{R}^{n_{x}}, \mathbf{x}^{L}$ and $\mathbf{x}^{U}$, such that $\mathbf{x}^{L} \leq \mathbf{x}(t, \mathbf{p}) \leq \mathbf{x}^{U}, \forall(t, \mathbf{p}) \in I \times P$. Since $\mathbf{x}$ is continuous and $I \times P$ is compact, such vectors certainly exist. Let $\mathbf{c}^{0}(t, \mathbf{p})=\mathbf{x}^{L}$ and $\mathbf{C}^{0}(t, \mathbf{p})=$ 
$\mathbf{x}^{U}, \forall(t, \mathbf{p}) \in I \times P$. Now consider the successive approximations defined recursively by

$$
\begin{gathered}
\mathbf{c}^{k+1}(t, \mathbf{p})=\mathbf{c}_{0}(\mathbf{p})+\int_{t_{0}}^{t} \mathbf{u}\left(s, \mathbf{p}, \mathbf{c}^{k}(s, \mathbf{p}), \mathbf{C}^{k}(s, \mathbf{p})\right) d s \\
\mathbf{C}^{k+1}(t, \mathbf{p})=\mathbf{C}_{0}(\mathbf{p})+\int_{t_{0}}^{t} \mathbf{o}\left(s, \mathbf{p}, \mathbf{c}^{k}(s, \mathbf{p}), \mathbf{C}^{k}(s, \mathbf{p})\right) d s .
\end{gathered}
$$

Note that $\mathbf{u}$ and $\mathbf{o}$ are defined on $I \times P \times \mathbb{R}^{n_{x}} \times \mathbb{R}^{n_{x}}$ and Lipschitz on all of $\mathbb{R}^{n_{x}} \times \mathbb{R}^{n_{x}}$ uniformly on $I \times P$ by Assumption 4.1. Thus, Theorem A.1 in Appendix A may be applied to (2), which proves that the successive approximations $\mathbf{c}^{k}$ and $\mathbf{C}^{k}$ in (3) exist and converge uniformly to the unique solutions of (2), $\mathbf{c}$ and $\mathbf{C}$, on $I \times P$.

Next, note that $\mathbf{c}^{0}(t, \cdot)$ and $\mathbf{C}^{0}(t, \cdot)$ are trivially convex and concave relaxations of $\mathbf{x}(t, \cdot)$ on $P$, respectively, for each fixed $t \in I$. Suppose that the same is true of $\mathbf{c}^{k}$ and $\mathbf{C}^{k}$. Then, by Definition 4.2, $\mathbf{u}\left(t, \cdot, \mathbf{c}^{k}(t, \cdot), \mathbf{C}^{k}(t, \cdot)\right)$ and $\mathbf{o}\left(t, \cdot, \mathbf{c}^{k}(t, \cdot), \mathbf{C}^{k}(t, \cdot)\right)$ are, respectively, convex and concave relaxations of $\mathbf{f}(t, \cdot, \mathbf{x}(t, \cdot))$ on $P$, for every fixed $t \in I$. Combining this with integral monotonicity,

$$
\begin{aligned}
\int_{t_{0}}^{t} \mathbf{u}\left(s, \mathbf{p}, \mathbf{c}^{k}(s, \mathbf{p}), \mathbf{C}^{k}(s, \mathbf{p})\right) d s & \leq \int_{t_{0}}^{t} \mathbf{f}(s, \mathbf{p}, \mathbf{x}(s, \mathbf{p})) d s \\
& \leq \int_{t_{0}}^{t} \mathbf{o}\left(s, \mathbf{p}, \mathbf{c}^{k}(s, \mathbf{p}), \mathbf{C}^{k}(s, \mathbf{p})\right) d s
\end{aligned}
$$

for all $(t, \mathbf{p}) \in I \times P$. But since $\mathbf{c}_{0}(\mathbf{p}) \leq \mathbf{x}_{0}(\mathbf{p}) \leq \mathbf{C}_{0}(\mathbf{p})$ for all $\mathbf{p} \in P$, (3) shows that

$$
\mathbf{c}^{k+1}(t, \mathbf{p}) \leq \mathbf{x}_{0}(\mathbf{p})+\int_{t_{0}}^{t} \mathbf{f}(s, \mathbf{p}, \mathbf{x}(s, \mathbf{p})) d s \leq \mathbf{C}^{k+1}(t, \mathbf{p}), \quad \forall(t, \mathbf{p}) \in I \times P,
$$

which, by the integral form of (1), gives

$$
\mathbf{c}^{k+1}(t, \mathbf{p}) \leq \mathbf{x}(t, \mathbf{p}) \leq \mathbf{C}^{k+1}(t, \mathbf{p}), \quad \forall(t, \mathbf{p}) \in I \times P .
$$

Theorem 2.1 proves that

$$
\int_{t_{0}}^{t} \mathbf{u}\left(s, \cdot, \mathbf{c}^{k}(s, \cdot), \mathbf{C}^{k}(s, \cdot)\right) d s \text { and } \int_{t_{0}}^{t} \mathbf{o}\left(s, \cdot, \mathbf{c}^{k}(s, \cdot), \mathbf{C}^{k}(s, \cdot)\right) d s
$$

are, respectively, convex and concave on $P$, for every fixed $t \in I$. Since $\mathbf{c}_{0}$ and $\mathbf{C}_{0}$ are respectively convex and concave by hypothesis, (3) shows that $\mathbf{c}^{k+1}$ and $\mathbf{C}^{k+1}$ are, respectively, convex and concave on $P$ for every fixed $t \in I$. Therefore, by induction, $\mathbf{c}^{k}(t, \cdot)$ and $\mathbf{C}^{k}(t, \cdot)$ are, respectively, convex and concave relaxations of $\mathbf{x}(t, \cdot)$ on $P$, for each fixed $t \in I$ and every $k \in \mathbb{N}$.

It was shown above that, as $k \rightarrow \infty, \mathbf{c}^{k}$ and $\mathbf{C}^{k}$ converge uniformly to the unique solutions of (2) on $I \times P$. Then, taking limits, it is clear that $\mathbf{c}(t, \cdot)$ and $\mathbf{C}(t, \cdot)$ are, respectively, convex and concave relaxations of $\mathbf{x}(t, \cdot)$ on $P$, for each fixed $t \in I$.

The preceding theorem shows that convex and concave relaxations of the solutions of the parametric ordinary differential equations (1) can be constructed by simply integrating any C-system of the form (2). Moreover, note that McCormick's composition rule and factorable representation 
[11] may be used to generate relaxations of general functions of $\mathbf{x}$ based on $\mathbf{c}$ and $\mathbf{C}$, so that the previous results can be used to generate convex and concave relaxations for very general optimal control problems.

\subsection{Convergence properties}

A major motivation for the construction of convex and concave relaxations is their use in spatial branch-and-bound algorithms for global optimization. For this application, convex and/or concave relaxations valid on subintervals of the feasible set are used to generate a convergent sequence of upper and lower bounds on the minimum or maximum of a given function. For a relaxation scheme to be useful in this regard, it is necessary to ensure that the relaxations being used lead to a bounding operation which is consistent (see Definition IV.4, [7]). This property is required for the resulting branch-and-bound algorithm to be finite $\varepsilon$-convergent. Essentially, for a consistent bounding operation to be possible, the relaxations must converge as the parameter set $P$ is partitioned, and must achieve the original function value in the limit as $P$ tends toward degeneracy. The first theorem below demonstrates these properties for the relaxations $\mathbf{c}$ and $\mathbf{C}$, and the second establishes that this convergence is in fact monotonic. As mentioned previously, McCormick's composition rule and factorable representation [11] may be used to generate relaxations of general functions of $\mathbf{x}$ using $\mathbf{c}$ and $\mathbf{C}$, so the results below can be used to establish consistency for very general optimal control problems.

In this section, closed $n_{p}$-dimensional subintervals of $P$ are denoted by $P^{\ell}$ (sometimes $P^{*}$ ), and for any $\mathbf{p} \in P$, the interval $[\mathbf{p}, \mathbf{p}]$ denotes the singleton $\{\mathbf{p}\}$ and is called a degenerate interval. Note that the results of the previous sections all remain true if some subinterval $P^{\ell} \subset P$ is considered in place of $P$. Thus, it is sensible to define the functions $\mathbf{c}^{\ell}$ and $\mathbf{C}^{\ell}$ as the relaxations of $\mathbf{x}$ constructed over some subinterval $P^{\ell}$. Analogously, it is sensible to refer to the initial condition functions, $\mathbf{c}_{0}^{\ell}$ and $\mathbf{C}_{0}^{\ell}$, and the right-hand sides, $\mathbf{u}^{\ell}$ and $\mathbf{o}^{\ell}$, all defined as before with $P^{\ell}$ in place of $P$. Using this notation, consider the auxiliary system of ODEs

$$
\begin{aligned}
\dot{\mathbf{c}}^{\ell}(t, \mathbf{p}) & =\mathbf{u}^{\ell}\left(t, \mathbf{p}, \mathbf{c}^{\ell}(t, \mathbf{p}), \mathbf{C}^{\ell}(t, \mathbf{p})\right), & \mathbf{c}^{\ell}\left(t_{0}, \mathbf{p}\right) & =\mathbf{c}_{0}^{\ell}(\mathbf{p}), \\
\dot{\mathbf{C}}^{\ell}(t, \mathbf{p}) & =\mathbf{o}^{\ell}\left(t, \mathbf{p}, \mathbf{c}^{\ell}(t, \mathbf{p}), \mathbf{C}^{\ell}(t, \mathbf{p})\right), & \mathbf{C}^{\ell}\left(t_{0}, \mathbf{p}\right) & =\mathbf{C}_{0}^{\ell}(\mathbf{p}),
\end{aligned}
$$

for any $P^{\ell} \subset P$. In the remainder of this section, we consider a procedure which, given any subinterval $P^{\ell} \subset P$, furnishes a C-system of (1) on $P^{\ell}$ of the form (4). The following properties of the state relaxations $\mathbf{c}^{\ell}$ and $\mathbf{C}^{\ell}$ are of primary interest.

\section{Definition 4.3}

A procedure for generating state relaxations of (1), $\mathbf{c}^{\ell}$ and $\mathbf{C}^{\ell}$, is partition convergent if, for any nested sequence of subintervals $\left\{P^{\ell}\right\} \rightarrow P^{*},\left\{\mathbf{c}^{\ell}\right\} \rightarrow \mathbf{c}^{*}$ and $\left\{\mathbf{C}^{\ell}\right\} \rightarrow \mathbf{C}^{*}$ uniformly on $I \times P^{*}$. Furthermore, a procedure for generating state relaxations is degenerate perfect if the condition $P^{*}=[\mathbf{p}, \mathbf{p}]$ for some $\mathbf{p} \in P$ implies that $\mathbf{c}^{*}(\cdot, \mathbf{p})=\mathbf{x}(\cdot, \mathbf{p})=\mathbf{C}^{*}(\cdot, \mathbf{p})$.

\section{Definition 4.4}

A procedure for generating state relaxations of (1), $\mathbf{c}^{\ell}$ and $\mathbf{C}^{\ell}$, is partition monotonic if, for any subintervals $P^{2} \subset P^{1} \subset P, \mathbf{c}^{2}(t, \mathbf{p}) \geq \mathbf{c}^{1}(t, \mathbf{p})$ and $\mathbf{C}^{2}(t, \mathbf{p}) \leq \mathbf{C}^{1}(t, \mathbf{p})$ for all $(t, \mathbf{p}) \in I \times P^{2}$. 
The following two definitions define properties of C-systems which are analogous to the properties of state relaxations described in Definitions 4.3 and 4.4. In fact, the main results of this section show that if a procedure for generating C-systems of the form (4) satisfies these properties, then generating the state relaxations $\mathbf{c}^{\ell}$ and $\mathbf{C}^{\ell}$ as the solutions of (4) is a partition convergent, degenerate perfect and partition monotonic procedure. Again, functions satisfying these conditions can be constructed automatically by the procedure described in $\S 5$.

\section{Definition 4.5}

A procedure for generating C-systems of (1) is partition convergent if, for any nested sequence of subintervals $\left\{P^{\ell}\right\} \rightarrow P^{*}$, the C-systems (4) satisfy

1. $\left\{\mathbf{c}_{0}^{\ell}\right\} \rightarrow \mathbf{c}_{0}^{*}$ and $\left\{\mathbf{C}_{0}^{\ell}\right\} \rightarrow \mathbf{C}_{0}^{*}$ uniformly on $P^{*}$, and

2. $\left\{\mathbf{u}^{\ell}\right\} \rightarrow \mathbf{u}^{*}$ and $\left\{\mathbf{o}^{\ell}\right\} \rightarrow \mathbf{o}^{*}$ uniformly on $I \times P^{*} \times \mathbb{R}^{n_{x}} \times \mathbb{R}^{n_{x}}$.

Furthermore, a procedure for generating C-systems is degenerate perfect if the condition $P^{*}=$ $[\mathbf{p}, \mathbf{p}]$ for some $\mathbf{p} \in P$ implies that

1. $\mathbf{c}_{0}^{*}(\mathbf{p})=\mathbf{x}_{0}(\mathbf{p})=\mathbf{C}_{0}^{*}(\mathbf{p})$, and

2. $\mathbf{u}^{*}(t, \mathbf{p}, \mathbf{z}, \mathbf{y})=\mathbf{f}(t, \mathbf{p}, \mathbf{x}(t, \mathbf{p}))=\mathbf{o}^{*}(t, \mathbf{p}, \mathbf{z}, \mathbf{y}), \quad \forall(t, \mathbf{z}, \mathbf{y}) \in I \times \mathbb{R}^{n_{x}} \times \mathbb{R}^{n_{x}}$.

\section{Definition 4.6}

A procedure for generating C-systems of (1) is partition monotonic if, for any $P^{2} \subset P^{1} \subset P$, the C-systems (4) satisfy

1. $\mathbf{c}_{0}^{1}(\mathbf{p}) \leq \mathbf{c}_{0}^{2}(\mathbf{p})$ and $\mathbf{C}_{0}^{2}(\mathbf{p}) \leq \mathbf{C}_{0}^{1}(\mathbf{p}), \quad \forall \mathbf{p} \in P^{2}$, and

2. for any $\left(t, \mathbf{p}, \mathbf{z}^{1}, \mathbf{y}^{1}, \mathbf{z}^{2}, \mathbf{y}^{2}\right) \in I \times P^{2} \times \mathbb{R}^{4 n_{x}}$, the inequalities

$$
\mathbf{u}^{1}\left(t, \mathbf{p}, \mathbf{z}^{1}, \mathbf{y}^{1}\right) \leq \mathbf{u}^{2}\left(t, \mathbf{p}, \mathbf{z}^{2}, \mathbf{y}^{2}\right) \quad \text { and } \quad \mathbf{o}^{2}\left(t, \mathbf{p}, \mathbf{z}^{2}, \mathbf{y}^{2}\right) \leq \mathbf{o}^{1}\left(t, \mathbf{p}, \mathbf{z}^{1}, \mathbf{y}^{1}\right)
$$

hold, provided that $\mathbf{z}^{1} \leq \mathbf{z}^{2} \leq \mathbf{x}(t, \mathbf{p}) \leq \mathbf{y}^{2} \leq \mathbf{y}^{1}$.

\section{Theorem 4.2}

If the $\mathrm{C}$-systems (4) are generated by a procedure which is partition convergent, then generating state relaxations of (1), $\mathbf{c}^{\ell}$ and $\mathbf{C}^{\ell}$, as the solutions of (4) is a partition convergent procedure. Furthermore, if the C-systems (4) are generated by a procedure which is degenerate perfect, then generating state relaxations of (1), $\mathbf{c}^{\ell}$ and $\mathbf{C}^{\ell}$, as the solutions of (4) is a degenerate perfect procedure.

Proof

Consider any nested and convergent sequence of subintervals of $P,\left\{P^{\ell}\right\} \rightarrow P^{*}$. Using the conditions of Definition 4.5, the uniform convergence of $\left\{\mathbf{c}^{\ell}\right\}$ and $\left\{\mathbf{C}^{\ell}\right\}$ to $\mathbf{c}^{*}$ and $\mathbf{C}^{*}$, respectively, on $I \times P^{*}$ is given by Lemma A.1 in Appendix A.

With $P^{*}=[\mathbf{p}, \mathbf{p}]$, the above argument shows that $\left\{\mathbf{c}^{\ell}\right\}$ and $\left\{\mathbf{C}^{\ell}\right\}$ converge uniformly on $I \times[\mathbf{p}, \mathbf{p}]$, and the limiting functions are $\mathbf{c}^{*}$ and $\mathbf{C}^{*}$. But, in this case,

$$
\begin{aligned}
\mathbf{c}^{*}(t, \mathbf{p}) & =\mathbf{c}_{0}^{*}(\mathbf{p})+\int_{t_{0}}^{t} \mathbf{u}^{*}\left(s, \mathbf{p}, \mathbf{c}^{*}(s, \mathbf{p}), \mathbf{C}^{*}(s, \mathbf{p})\right) d s, \\
& =\mathbf{x}_{0}(\mathbf{p})+\int_{t_{0}}^{t} \mathbf{f}(s, \mathbf{p}, \mathbf{x}(s, \mathbf{p})) d s=\mathbf{x}(t, \mathbf{p}),
\end{aligned}
$$


for all $t \in I$. Of course, an analogous argument holds for $\mathbf{C}^{*}$.

\section{Theorem 4.3}

If the C-systems (4) are generated by a procedure which is partition monotonic, then generating state relaxations of (1), $\mathbf{c}^{\ell}$ and $\mathbf{C}^{\ell}$, as the solutions of (4) is a partition monotonic procedure.

Proof

Consider any $P^{2} \subset P^{1} \subset P$. Combining Condition 1 of Definition 4.6 with the fact that (4) is a C-system of (1) on $P^{\ell}$ for $\ell=2$, the following inequalities hold:

$$
\mathbf{c}_{0}^{1}(\mathbf{p}) \leq \mathbf{c}_{0}^{2}(\mathbf{p}) \leq \mathbf{x}_{0}(\mathbf{p}) \leq \mathbf{C}_{0}^{2}(\mathbf{p}) \leq \mathbf{C}_{0}^{1}(\mathbf{p}), \quad \forall \mathbf{p} \in P^{2}
$$

Further, $\mathbf{c}_{0}^{2}$ and $\mathbf{C}_{0}^{2}$ are, respectively, convex and concave relaxations of $\mathbf{x}_{0}$ on $P^{2}$. It will be shown that

$$
\mathbf{c}^{1}(t, \mathbf{p}) \leq \mathbf{c}^{2}(t, \mathbf{p}) \leq \mathbf{x}(t, \mathbf{p}) \leq \mathbf{C}^{2}(t, \mathbf{p}) \leq \mathbf{C}^{1}(t, \mathbf{p}), \quad \forall(t, \mathbf{p}) \in I \times P^{2} .
$$

This inequality is shown by comparing the successive approximations for $\mathbf{c}^{\ell}$ and $\mathbf{C}^{\ell}, \ell \in\{1,2\}$. These are defined analogously to (3) and denoted by $\mathbf{c}^{k, \ell}$ and $\mathbf{C}^{k, \ell}$, respectively. As in the proof of Theorem 4.1, we may choose two vectors $\mathbf{x}^{L}$ and $\mathbf{x}^{U}$ such that $\mathbf{x}^{L} \leq \mathbf{x}(t, \mathbf{p}) \leq \mathbf{x}^{U}, \forall(t, \mathbf{p}) \in$ $I \times P^{1}$, and let $\mathbf{c}^{0, \ell}(t, \mathbf{p})=\mathbf{x}^{L}$ and $\mathbf{C}^{0, \ell}(t, \mathbf{p})=\mathbf{x}^{U}, \forall(t, \mathbf{p}) \in I \times P^{\ell}, \ell \in\{1,2\}$. As shown in the proof of Theorem 4.1, the successive approximations of (4), for both $\ell=1$ and $\ell=2$, exist and converge uniformly to the unique solutions on $I \times P^{2}$.

With these definitions, it is clear that $\mathbf{c}^{0, \ell}(t, \cdot)$ and $\mathbf{C}^{0, \ell}(t, \cdot)$ are, respectively, convex relaxations of $\mathbf{x}(t, \cdot)$ on $P^{\ell}$ for each $t \in I$ and both $\ell \in\{1,2\}$, and that

$$
\mathbf{c}^{k, 1}(t, \mathbf{p}) \leq \mathbf{c}^{k, 2}(t, \mathbf{p}) \leq \mathbf{x}(t, \mathbf{p}) \leq \mathbf{C}^{k, 2}(t, \mathbf{p}) \leq \mathbf{C}^{k, 1}(t, \mathbf{p}), \quad \forall(t, \mathbf{p}) \in I \times P^{2},
$$

for $k=0$. Assume that these observations hold for some arbitrary $k \in \mathbb{N}$. Then the definition of the successive approximations, Conditions 1 and 2 of Definition 4.6, and integral monotonicity ensure that $\mathbf{c}^{k+1,1}(t, \mathbf{p}) \leq \mathbf{c}^{k+1,2}(t, \mathbf{p})$ and $\mathbf{C}^{k+1,2}(t, \mathbf{p}) \leq \mathbf{C}^{k+1,1}(t, \mathbf{p})$ for all $(t, \mathbf{p}) \in I \times P^{2}$. Similarly, Condition 2 of Definition 4.2 implies that $\mathbf{c}^{k+1,2}(t, \cdot)$ and $\mathbf{C}^{k+1,2}(t, \cdot)$ are, respectively, convex and concave relaxations of $\mathbf{x}(t, \cdot)$ on $P^{2}$, for each fixed $t \in I$. Thus, the desired inequalities and relaxation properties have been recovered for the $(k+1)^{s t}$ successive approximations, so that induction over $k$ and the uniform convergence of the successive approximations guarantees (6).

\section{COMPUTING STATE RELAXATIONS}

According to Theorem 4.1, state relaxations for (1) on $I \times P$ can be computed by constructing any C-system of (1) and solving it numerically. In [19], the authors developed a method for automatically generating $\mathrm{C}$-system using only the computational graphs of the functions $\mathbf{f}$ and $\mathrm{x}_{0}$. Combined, these developments provide a means to compute guaranteed convex and concave relaxations for the 
parametric solutions of arbitrary nonlinear ODEs. For completeness, the automatic generation of Csystems is described in $\S 5.2$ below. This procedure makes use of McCormick's relaxation technique, which is described in the following section.

\subsection{McCormick relaxations}

McCormick's relaxation technique applies to factorable functions. Roughly speaking, a function is factorable if it can be defined by the finite recursive application of binary additions, binary multiplications and composition with a pre-defined library of univariate functions, typically including exponential and logarithmic functions, square root, odd and even integer powers, trigonometric functions, etc. Letting $\mathcal{E}$ denote this collection of functions, we have the following definition.

\section{Definition 5.1}

Let $S \subset \mathbb{R}^{n_{p}}$ and $\mathcal{F}: S \rightarrow \mathbb{R}$. $\mathcal{F}$ is factorable if it can be expressed in terms of a finite number of factors $v_{1}, \ldots, v_{m}: S \rightarrow \mathbb{R}$ such that, given $\mathbf{p} \in S, v_{i}(\mathbf{p})=p_{i}$ for $i=1, \ldots, n_{p}$, and $v_{k}$ is defined for each $n_{p}<k \leq m$ as either

(a) $v_{k}(\mathbf{p})=v_{i}(\mathbf{p})+v_{j}(\mathbf{p})$, with $i, j<k$, or

(b) $v_{k}(\mathbf{p})=v_{i}(\mathbf{p}) v_{j}(\mathbf{p})$, with $i, j<k$, or

(c) $v_{k}(\mathbf{p})=w\left(v_{i}(\mathbf{p})\right)$, with $i<k$ and $w \in \mathcal{E}$,

and $\mathcal{F}(\mathbf{p})=v_{m}(\mathbf{p})$. A vector function is called factorable if each element is factorable.

Nearly every function with a known computational graph is factorable. For any such function, convex and concave relaxations can be obtained on $P \equiv\left[\mathbf{p}^{L}, \mathbf{p}^{U}\right] \subset S$ by McCormick's relaxation technique. The method associates with each factor $v_{k}$ the quantities $\left(v_{k}^{L}, v_{k}^{U}, v_{k}^{c}, v_{k}^{C}\right)$, which are, respectively, lower and upper bounds for $v_{k}$ and convex and concave relaxations of $v_{k}$ on $P$. For a given $\mathbf{p} \in P$, the computation is initialized by letting $\left(v_{k}^{L}, v_{k}^{U}, v_{k}^{c}(\mathbf{p}), v_{k}^{C}(\mathbf{p})\right)=\left(p_{k}^{L}, p_{k}^{U}, p_{k}, p_{k}\right)$, for all $k \leq n_{p}$, and computing these values for the remaining factors recursively using known rules based on the definition of $v_{k}$ in Definition 5.1. Rules for addition, multiplication and composition with many common univariate functions $\left(e^{x}, \sin (x), x^{n},-x\right.$, etc.), as well as a detailed definition of McCormick's relaxation technique can be found in [11, 19].

\subsection{Automatic Construction of C-Systems}

In this section, it is shown that McCormick's relaxation technique can be used to construct functions $\mathbf{c}_{0}, \mathbf{C}_{0}, \mathbf{u}$ and $\mathbf{o}$ satisfying Definition 4.2. Clearly, $\mathbf{c}_{0}$ and $\mathbf{C}_{0}$ can be constructed by directly applying McCormick's technique to the function $\mathbf{x}_{0}$. In order to construct $\mathbf{u}$ and $\mathbf{o}$, a generalization of McCormick's technique is applied to the function $\mathrm{f}$. This requires state bounds, defined as follows.

Definition 5.2

Functions $\mathbf{x}^{L}, \mathbf{x}^{U}: I \rightarrow \mathbb{R}^{n_{x}}$ are called state bounds for $\mathbf{x}$ on $I \times P$ if $\mathbf{x}^{L}(t) \leq \mathbf{x}(t, \mathbf{p}) \leq \mathbf{x}^{U}(t)$, $\forall(t, \mathbf{p}) \in I \times P$.

\section{Assumption 5.1}

State bounds for $\mathrm{x}$ on $I \times P$ are available which are continuous on $I$ and satisfy $X(t) \equiv$ $\left[\mathbf{x}^{L}(t), \mathbf{x}^{U}(t)\right] \subset D, \forall t \in I$. 
Numerical techniques for generating state bounds may be found in $[30,31,14,32,33,34]$. When $n_{x}>1$ there may exist no interval which both encloses the image of $P$ under $\mathbf{x}(t, \cdot)$ for some $t \in I$ and is contained in $D$. However, this is rarely a problem in practical applications and Assumption 5.1 is typically not difficult to satisfy.

\section{Remark 5.1}

Of course, the state bounds $\mathrm{x}^{L}$ and $\mathrm{x}^{U}$ are trivially state relaxations for $\mathbf{x}$ on $I \times P$. However, interval bounds can be quite crude and cannot capture the parameter dependence of the solution $\mathrm{x}$. Indeed, all known methods for relaxing the solutions of nonlinear ODEs use state bounds at an intermediate stage in their computation.

Now consider the ODEs (1) and suppose that each $f_{i}$ is factorable. In [19], it was observed that McCormick's rules can be used to construct relaxations of composite functions of the form $f_{i}(t, \cdot, \mathbf{x}(t, \cdot))$ from known bounds and relaxations for $\mathbf{x}(t, \cdot)$. The construction is as follows. Choose some $i$ and let $v_{1}, \ldots, v_{m}: I \times P \times D \rightarrow \mathbb{R}$ be a factorable representation of $f_{i}$, where

$$
\begin{aligned}
& v_{1}(t, \mathbf{p}, \mathbf{z})=t, \\
& v_{k+1}(t, \mathbf{p}, \mathbf{z})=p_{k}, \quad \forall k \in\left\{1, \ldots, n_{p}\right\}, \\
& v_{k+n_{p}+1}(t, \mathbf{p}, \mathbf{z})=z_{k}, \quad \forall k \in\left\{1, \ldots, n_{x}\right\} .
\end{aligned}
$$

Define the functions $v_{1}^{c / C}, \ldots, v_{n_{x}+n_{p}+1}^{c / C}: I \times P \times \mathbb{R}^{n_{x}} \times \mathbb{R}^{n_{x}} \rightarrow \mathbb{R}$ for any $(t, \mathbf{p}, \boldsymbol{\phi}, \boldsymbol{\psi})$ by first assigning

$$
\begin{aligned}
& \left(v_{1}^{L}, v_{1}^{U}, \bar{v}_{1}^{c}, \bar{v}_{1}^{C}\right)=(t, t, t, t), \\
& \left(v_{k+1}^{L}, v_{k+1}^{U}, \bar{v}_{k+1}^{c}, \bar{v}_{k+1}^{C}\right)=\left(p_{k}^{L}, p_{k}^{U}, p_{k}, p_{k}\right), \quad \forall k \in\left\{1, \ldots, n_{p}\right\}, \\
& \left(v_{k+n_{p}+1}^{L}, v_{k+n_{p}+1}^{U}, \bar{v}_{k+n_{p}+1}^{c}, \bar{v}_{k+n_{p}+1}^{C}\right)=\left(x_{k}^{L}(t), x_{k}^{U}(t), \phi_{k}, \psi_{k}\right), \quad \forall k \in\left\{1, \ldots, n_{x}\right\},
\end{aligned}
$$

where the argument lists have been omitted for brevity, second, making the assignments

$$
\begin{aligned}
v_{k}^{c}(t, \mathbf{p}, \boldsymbol{\phi}, \boldsymbol{\psi}) & =\operatorname{mid}\left(v_{k}^{L}, v_{k}^{U}, \bar{v}_{k}^{c}(t, \mathbf{p}, \boldsymbol{\phi}, \boldsymbol{\psi})\right), \\
v_{k}^{C}(t, \mathbf{p}, \boldsymbol{\phi}, \boldsymbol{\psi}) & =\operatorname{mid}\left(v_{k}^{L}, v_{k}^{U}, \bar{v}_{k}^{C}(t, \mathbf{p}, \boldsymbol{\phi}, \boldsymbol{\psi})\right),
\end{aligned}
$$

for all $k \in\left\{1, \ldots, n_{x}+n_{p}+1\right\}$, and finally, computing $\left(v_{k}^{L}, v_{k}^{U}, v_{k}^{c}, v_{k}^{C}\right)$ for $n_{p}+n_{x}+1<k \leq m$ by recursive application of McCormick's relaxation rules.

Now, define the functions $u_{i}, o_{i}: I \times P \times \mathbb{R}^{n_{x}} \times \mathbb{R}^{n_{x}} \rightarrow \mathbb{R}$ for any $(t, \mathbf{p}, \phi, \psi)$ by letting

$$
u_{i}(t, \mathbf{p}, \boldsymbol{\phi}, \boldsymbol{\psi}) \equiv v_{m}^{c}(t, \mathbf{p}, \boldsymbol{\phi}, \boldsymbol{\psi}) \quad \text { and } \quad o_{i}(t, \mathbf{p}, \boldsymbol{\phi}, \boldsymbol{\psi}) \equiv v_{m}^{C}(t, \mathbf{p}, \boldsymbol{\phi}, \boldsymbol{\psi})
$$

Suppose that $\phi(t, \cdot)$ and $\psi(t, \cdot)$ are convex and concave relaxations of $\mathbf{x}(t, \cdot)$ on $P$, respectively, for some $t \in I$. Recalling that $\mathbf{x}(t, \mathbf{p}) \in\left[\mathbf{x}^{L}(t), \mathbf{x}^{U}(t)\right], \forall \mathbf{p} \in P$, this implies that

$$
\begin{aligned}
& \operatorname{mid}\left(\mathbf{x}^{L}(t), \mathbf{x}^{U}(t), \boldsymbol{\phi}(t, \cdot)\right)=\max \left(\mathbf{x}^{L}(t), \boldsymbol{\phi}(t, \cdot)\right), \\
& \operatorname{mid}\left(\mathbf{x}^{L}(t), \mathbf{x}^{U}(t), \boldsymbol{\psi}(t, \cdot)\right)=\min \left(\mathbf{x}^{U}(t), \boldsymbol{\psi}(t, \cdot)\right),
\end{aligned}
$$


are also convex and concave relaxations of $\mathbf{x}(t, \cdot)$ on $P$, respectively. Then, since $\mathbf{u}$ and $\mathbf{o}$ are defined by recursive application of McCormick's relaxation rules, it follows by an inductive argument [19] that $u_{i}(t, \cdot, \phi(t, \cdot), \boldsymbol{\psi}(t, \cdot))$ and $o_{i}(t, \cdot, \phi(t, \cdot), \boldsymbol{\psi}(t, \cdot))$ are, respectively, convex and concave relaxations of $f_{i}(t, \cdot, \mathbf{x}(t, \cdot))$ on $P$. In general, this construction guarantees that $\mathbf{u}$ and $\mathbf{o}$ satisfy Condition 2 Definition 4.2. The reader is referred to [19] for a detailed description of this procedure and formal proofs (see in particular Theorem 14).

It is also shown in $\S 7.2$ of [19] that the functions $\mathbf{u}$ and $\mathbf{o}$ are continuous on $I \times P \times \mathbb{R}^{n_{x}} \times$ $\mathbb{R}^{n_{x}}$ and satisfy the global Lipschitz condition of Assumption 4.1, provided that the factorable representation of $\mathbf{f}$ satisfies some mild conditions. It is worth noting that these conditions do not imply a global Lipschitz condition on $\mathbf{f}$, but they do imply the local condition of Assumption 3.1. Essentially, for fixed $(t, \mathbf{p}) \in I \times P$, the global Lipschitz condition on $\mathbf{u}$ and $\mathbf{o}$ is made possible by the state bounds $X(t)$. As outlined above, the construction of these functions involves mapping any arguments $(\phi, \psi) \in \mathbb{R}^{n_{x}} \times \mathbb{R}^{n_{x}}$ into $X(t) \times X(t)$ in a Lipschitz manner (using the mid function), so that Lipschitz continuity of $\mathbf{u}(t, \mathbf{p}, \cdot, \cdot)$ and $\mathbf{o}(t, \mathbf{p}, \cdot, \cdot)$ need only hold on this compact interval [19].

Finally, it is shown in $\S 7.3$ of [19] that constructing $\mathbf{c}_{0}, \mathbf{C}_{0}, \mathbf{u}$ and $\mathbf{o}$ as described above is a partition convergent, degenerate perfect and partition monotonic procedure as per Definitions 4.5 and 4.6. It then follows from Theorems 4.2 and 4.3 that the resulting state relaxations are partition convergent, degenerate perfect and partition monotonic.

\subsection{Implementation}

This section describes the computational implementation of the nonlinear state relaxation theory developed in this article. To compute state bounds, the method in [30] is used, which describes $\mathbf{x}^{L}$ and $\mathbf{x}^{U}$ as the solutions of another auxiliary system of ODEs. Given $\left(t_{f}, \mathbf{p}\right) \in I \times P$ at which the values $\mathbf{c}\left(t_{f}, \mathbf{p}\right)$ and $\mathbf{C}\left(t_{f}, \mathbf{p}\right)$ are desired, the ODEs describing the state bounds are numerically integrated simultaneously with the system (2) at $\mathbf{p}$, from $t_{0}$ to $t_{f}$. Numerical simulation of (2) is done using CVODE [29] with relative and absolute tolerances of $1 \times 10^{-8}$. To begin this computation, the initial conditions $\mathbf{c}_{0}(\mathbf{p})$ and $\mathbf{C}_{0}(\mathbf{p})$ are computed by taking standard McCormick relaxations of $\mathbf{x}_{0}$ on $P$, evaluated at $\mathbf{p}$. This is done using the $\mathrm{C}++$ library $\mathrm{MC}++$, which automatically computes interval extensions and McCormick relaxations of factorable functions using operator overloading (http://www3.imperial.ac.uk/people/b.chachuat/research). MC++ is the successor of $1 \mathrm{ibMC}$, which is described in detail in [15]. Whenever it is required to evaluate the right-hand side of (2), the functions $u_{i}$ and $o_{i}$ are evaluated automatically using $\mathrm{MC}++$, by initializing the computation of McCormick relaxations as in (8) in $\S 5.2$.

\section{SAMPLE PROBLEM}

\section{Example 6.1}

Section 1.2.4 of [26] discusses a negative resistance circuit consisting of an inductor, a capacitor and a resistive element in parallel. The circuit can be described by the nonlinear ODEs

$$
\dot{x}_{1}=\frac{1}{L} x_{2}, \quad \dot{x}_{2}=-\frac{1}{C}\left[x_{1}-x_{2}+\frac{1}{3} x_{2}^{3}\right],
$$




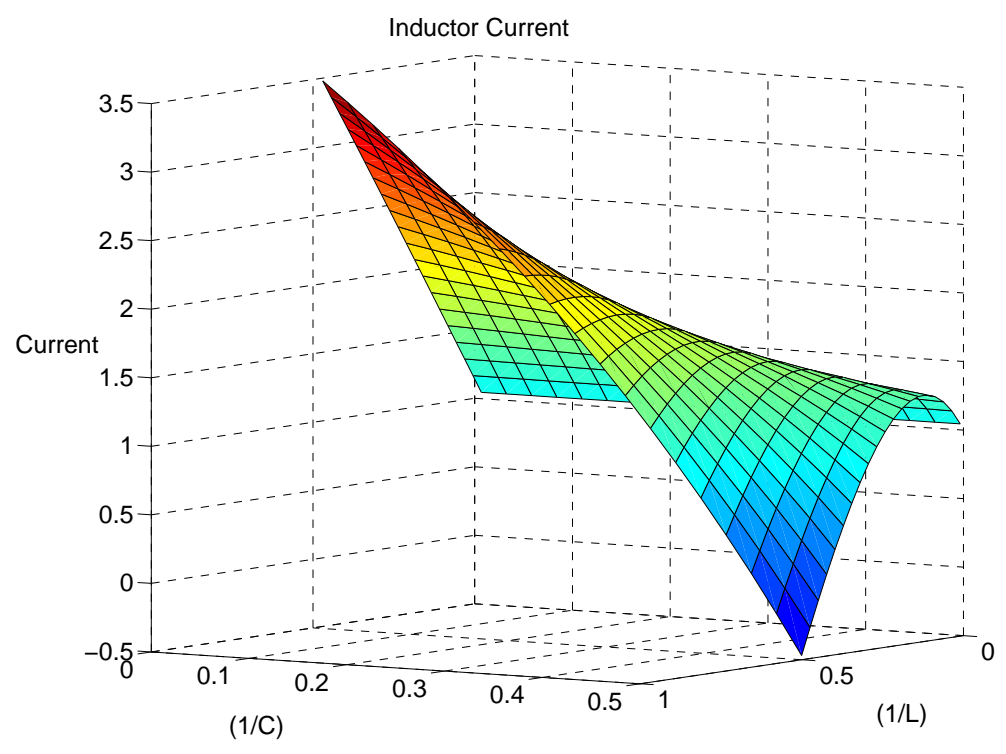

Figure 1. The parametric final time solution of the ODEs $(10), x_{1}\left(t_{f}, \cdot\right)$, on the interval $P=[0.01,0.5]^{2}$.

where $L$ and $C$ are the inductance and capacitance respectively, $x_{1}$ is the current through the inductor, and $x_{2}$ is the voltage across the capacitor. It is assumed that time, $C, L, x_{1}$ and $x_{2}$ are scaled so that the equations above are dimensionless and all quantities are of order one with the possible exception of $(1 / L)$ and $(1 / C)$. Therefore, the initial value problem with $x_{0,1}=x_{0,2}=1$, $t_{0}=0$ and $t_{f}=5$ is considered. Letting the parameters be $p_{1}=(1 / C)$ and $p_{2}=(1 / L)$, the solution $x_{1}\left(t_{f}, \cdot\right)$ on the set $P=\left[p_{1}^{L}, p_{1}^{U}\right] \times\left[p_{2}^{L}, p_{2}^{U}\right]=[0.01,0.5] \times[0.01,0.5]$ is shown in Figure 1. The parametric final time solution is clearly nonconvex, with a single maximum at $\left(p_{1}, p_{2}\right)=(0.01,0.5)$ and two local minima, the global minimum at $\left(p_{1}, p_{2}\right)=(0.5,0.5)$, and a suboptimal local minimum at $\left(p_{1}, p_{2}\right)=(0.01,0.01)$.

Beginning from the functions

$$
\mathbf{x}_{0}=[1,1]^{\mathrm{T}}, \quad \mathbf{f}=\left[f_{1}, f_{2}\right]^{\mathrm{T}}=\left[p_{1} x_{2}, \quad-p_{2}\left(x_{1}-x_{2}+\frac{1}{3} x_{2}^{3}\right)\right]^{\mathrm{T}},
$$

we need to construct functions $\mathbf{c}_{0}, \mathbf{C}_{0}, \mathbf{u}$ and $\mathbf{o}$ such that the auxiliary system (2) is a Csystem of (10) on $P$. Since $\mathbf{x}_{0}$ is constant, appropriate convex and concave relaxations are simply $\mathbf{c}_{0}=\mathbf{C}_{0}=\mathbf{x}_{0}$.

Now, consider $f_{1}$. For any $(t, \mathbf{p}, \mathbf{z}, \mathbf{y}) \in I \times P \times \mathbb{R}^{n_{x}} \times \mathbb{R}^{n_{x}}$, appropriate values for the functions $u_{1}$ and $o_{1}$ at $(t, \mathbf{p}, \mathbf{z}, \mathbf{y})$ can be computed by evaluating the McCormick convex and concave relaxations [11] of $f_{1}(t, \cdot, \cdot)$ over the interval $P \times X(t)$, with values for convex and concave relaxations of the state variables at $\mathbf{p}$ specified as $\operatorname{mid}\left(\mathbf{x}^{L}(t), \mathbf{x}^{U}(t), \mathbf{z}\right)$ and $\operatorname{mid}\left(\mathbf{x}^{L}(t), \mathbf{x}^{U}(t), \mathbf{y}\right)$, respectively. This is implemented by the factorization of $f_{1}$ shown in Table I with factors $v_{i}$, lower and upper bounds on each factor, $v_{i}^{L}$ and $v_{i}^{U}$, computed through standard interval arithmetic [27], and McCormick's convex and concave relaxation for each factor, $v^{c}$ and $v^{C}$. Note that the last two columns of Table I define $\bar{v}_{k}^{c}$ and $\bar{v}_{k}^{C}$, whereas subsequent factors are defined in terms of the values 
Table I. Factorization and computation of $f_{1}(t, \cdot, \cdot)$ at $(\mathbf{p}, \mathbf{x})$ and $u_{1}(t, \cdot, \cdot, \cdot)$ and $o_{1}(t, \cdot, \cdot, \cdot)$ at $(\mathbf{p}, \mathbf{z}, \mathbf{y})$.

\begin{tabular}{|c|c|c|c|c|c|}
\hline$i$ & $v_{i}$ & $v_{i}^{L}$ & $v_{i}^{U}$ & $\bar{v}_{i}^{c}$ & $\bar{v}_{i}^{C}$ \\
\hline 1 & $p_{1}$ & $p_{1}^{L}$ & $p_{1}^{U}$ & $p_{1}$ & $p_{1}$ \\
\hline 2 & $x_{2}$ & $x_{2}^{L}(t)$ & $x_{2}^{U}(t)$ & $\operatorname{mid}\left(x_{2}^{L}(t), x_{2}^{U}(t), z_{2}\right)$ & $\operatorname{mid}\left(x_{2}^{L}(t), x_{2}^{U}(t), y_{2}\right)$ \\
\hline 3 & $v_{1} v_{2}$ & $\min \{$ & $\max \{$ & $\max \{$ & $\min \{$ \\
& & $v_{1}^{L} v_{2}^{L}, v_{1}^{U} v_{2}^{U}$, & $v_{1}^{L} v_{2}^{L}, v_{1}^{U} v_{2}^{U}$, & $\alpha_{1}+\alpha_{2}-v_{2}^{L} v_{1}^{L}$, & $\gamma_{1}+\gamma_{2}-v_{2}^{L} v_{1}^{U}$, \\
& & $\left.v_{1}^{L} v_{2}^{U}, v_{1}^{U} v_{2}^{L}\right\}$ & $\left.v_{1}^{L} v_{2}^{U}, v_{1}^{U} v_{2}^{L}\right\}$ & $\left.\beta_{1}+\beta_{2}-v_{2}^{U} v_{1}^{U}\right\}$ & $\left.\delta_{1}+\delta_{2}-v_{2}^{U} v_{1}^{L}\right\}$ \\
\hline
\end{tabular}

$v_{k}^{c}$ and $v_{k}^{C}$. These quantities are related by the computations

$$
v_{k}^{c}=\max \left(\bar{v}_{k}^{c}, v_{k}^{L}\right) \quad \text { and } \quad v_{k}^{C}=\min \left(\bar{v}_{k}^{C}, v_{k}^{U}\right),
$$

which are omitted from Table I for simplicity. The quantities, $\alpha, \beta, \delta$ and $\gamma$ in the table are defined as

$$
\begin{aligned}
\alpha_{1}=\min \left\{v_{2}^{L} v_{1}^{c}, v_{2}^{L} v_{1}^{C}\right\}, & \alpha_{2}=\min \left\{v_{1}^{L} v_{2}^{c}, v_{1}^{L} v_{2}^{C}\right\}, \\
\beta_{1}=\min \left\{v_{2}^{U} v_{1}^{c}, v_{2}^{U} v_{1}^{C}\right\}, & \beta_{2}=\min \left\{v_{1}^{U} v_{2}^{c}, v_{1}^{U} v_{2}^{C}\right\}, \\
\gamma_{1}=\max \left\{v_{2}^{L} v_{1}^{c}, v_{2}^{L} v_{1}^{C}\right\}, & \gamma_{2}=\max \left\{v_{1}^{U} v_{2}^{c}, v_{1}^{U} v_{2}^{C}\right\}, \\
\delta_{1}=\max \left\{v_{2}^{U} v_{1}^{c}, v_{2}^{U} v_{1}^{C}\right\}, & \delta_{2}=\max \left\{v_{1}^{L} v_{2}^{c}, v_{1}^{L} v_{2}^{C}\right\} .
\end{aligned}
$$

The factorization of $f_{2}(t, \cdot, \cdot)$ is more complicated due to the cubic term. The convex and concave envelopes of the cubic function are known [35] and require the following definitions. Let $x_{2}^{\prime}$ and $x_{2}^{\prime \prime}$ be, respectively, the solutions of

$$
2\left(x_{2}^{\prime}\right)^{3}-3 x_{2}^{L}(t)\left(x_{2}^{\prime}\right)^{2}+\left(x_{2}^{L}(t)\right)^{3}=0 \quad \text { and } \quad 2\left(x_{2}^{\prime \prime}\right)^{3}-3 x_{2}^{U}(t)\left(x_{2}^{\prime \prime}\right)^{2}+\left(x_{2}^{U}(t)\right)^{3}=0,
$$

and define

$$
x_{2}^{*}=\left\{\begin{array}{cc}
x_{2}^{U}(t) \quad \text { if } \quad x_{2}^{U}(t) \leq 0 \\
x_{2}^{L}(t) \quad \text { if } \quad x_{2}^{L}(t) \geq 0 \\
x_{2}^{\prime} & \text { otherwise }
\end{array} \quad, \quad x_{2}^{* *}=\left\{\begin{array}{ccc}
x_{2}^{U}(t) & \text { if } \quad x_{2}^{U}(t) \leq 0 \\
x_{2}^{L}(t) & \text { if } x_{2}^{L}(t) \geq 0 \\
x_{2}^{\prime \prime} & \text { otherwise }
\end{array} .\right.\right.
$$

Further, define the functions

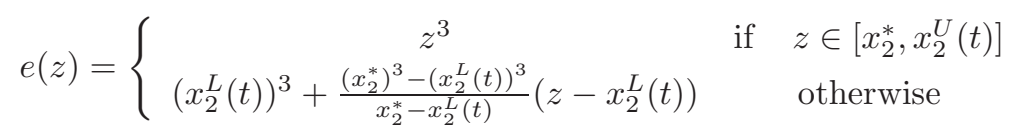

and

$$
E(z)=\left\{\begin{array}{ccc}
z^{3} & \text { if } & z \in\left[x_{2}^{L}(t), x_{2}^{* *}\right] \\
\left(x_{2}^{* *}\right)^{3}+\frac{\left(x_{2}^{U}(t)\right)^{3}-\left(x_{*}^{* *}\right)^{3}}{x_{2}^{U}(t)-x_{2}^{*}}\left(z-x_{2}^{* *}\right) & \text { otherwise }
\end{array} .\right.
$$

Now the factorization and McCormick relaxation of $f_{2}$ is given in Table II, again with values for convex and concave relaxations of the state variables at $\mathbf{p}$ specified as $\operatorname{mid}\left(\mathbf{x}^{L}(t), \mathbf{x}^{U}(t), \mathbf{z}\right)$ and 
Table II. Factorization and computation of $f_{2}(t, \cdot, \cdot)$ at $(\mathbf{p}, \mathbf{x})$ and $u_{2}(t, \cdot, \cdot, \cdot)$ and $o_{2}(t, \cdot, \cdot, \cdot)$ at $(\mathbf{p}, \mathbf{z}, \mathbf{y})$.

\begin{tabular}{|c|c|c|c|c|c|}
\hline$i$ & $v_{i}$ & $v_{i}^{L}$ & $v_{i}^{U}$ & $\bar{v}_{i}^{c}$ & $\bar{v}_{i}^{C}$ \\
\hline 1 & $p_{2}$ & $p_{2}^{L}$ & $p_{2}^{U}$ & $p_{2}$ & $p_{2}$ \\
\hline 2 & $x_{1}$ & $x_{1}^{L}(t)$ & $x_{1}^{U}(t)$ & $\operatorname{mid}\left(x_{1}^{L}(t), x_{1}^{U}(t), z_{1}\right)$ & $\operatorname{mid}\left(x_{1}^{L}(t), x_{1}^{U}(t), y_{1}\right)$ \\
\hline 3 & $x_{2}$ & $x_{2}^{L}(t)$ & $x_{2}^{U}(t)$ & $\operatorname{mid}\left(x_{2}^{L}(t), x_{2}^{U}(t), z_{2}\right)$ & $\operatorname{mid}\left(x_{2}^{L}(t), x_{2}^{U}(t), y_{2}\right)$ \\
\hline 4 & $v_{3}^{3}$ & $\left(v_{3}^{L}\right)^{3}$ & $\left(v_{3}^{U}\right)^{3}$ & $e\left(\operatorname{mid}\left(v_{3}^{c}, v_{3}^{C}, v_{3}^{L}\right)\right)$ & $E\left(\operatorname{mid}\left(v_{3}^{c}, v_{3}^{C}, v_{3}^{U}\right)\right)$ \\
\hline 5 & $(1 / 3) v_{4}$ & $(1 / 3) v_{4}^{L}$ & $(1 / 3) v_{4}^{U}$ & $(1 / 3) \operatorname{mid}\left(v_{4}^{c}, v_{4}^{C}, v_{4}^{L}\right)$ & $(1 / 3) \operatorname{mid}\left(v_{4}^{c}, v_{4}^{C}, v_{4}^{U}\right)$ \\
\hline 6 & $-v_{3}$ & $-v_{3}^{U}$ & $-v_{3}^{L}$ & $-\operatorname{mid}\left(v_{3}^{c}, v_{3}^{C}, v_{3}^{U}\right)$ & $-\operatorname{mid}\left(v_{3}^{c}, v_{3}^{C}, v_{3}^{L}\right)$ \\
\hline 7 & $v_{2}+v_{6}$ & $v_{2}^{L}+v_{6}^{L}$ & $v_{2}^{U}+v_{6}^{U}$ & $v_{2}^{c}+v_{6}^{c}$ & $v_{2}^{C}+v_{6}^{C}$ \\
\hline 8 & $v_{7}+v_{5}$ & $v_{7}^{L}+v_{5}^{L}$ & $v_{7}^{U}+v_{5}^{U}$ & $v_{7}^{c}+v_{5}^{c}$ & $v_{7}^{C}+v_{5}^{C}$ \\
\hline 9 & $-v_{1}$ & $-v_{1}^{U}$ & $-v_{1}^{L}$ & $-\operatorname{mid}\left(v_{1}^{c}, v_{1}^{C}, v_{1}^{U}\right)$ & $-\operatorname{mid}\left(v_{1}^{c}, v_{1}^{C}, v_{1}^{L}\right)$ \\
\hline 10 & $v_{8} v_{9}$ & $\begin{array}{c}\min \{ \\
v_{8}^{L} v_{9}^{L}, v_{8}^{U} v_{9}^{U} \\
\left.v_{8}^{L} v_{9}^{U}, v_{8}^{U} v_{9}^{L}\right\}\end{array}$ & $\begin{array}{c}\max \{ \\
v_{8}^{L} v_{9}^{L}, v_{8}^{U} v_{9}^{U} \\
\left.v_{8}^{L} v_{9}^{U}, v_{8}^{U} v_{9}^{L}\right\}\end{array}$ & $\begin{array}{c}\max \{ \\
\alpha_{8}+\alpha_{9}-v_{9}^{L} v_{8}^{L} \\
\left.\beta_{8}+\beta_{9}-v_{9}^{U} v_{8}^{U}\right\}\end{array}$ & $\begin{array}{c}\min \{ \\
\gamma_{8}+\gamma_{9}-v_{9}^{L} v_{8}^{U} \\
\left.\delta_{8}+\delta_{9}-v_{9}^{U} v_{8}^{L}\right\}\end{array}$ \\
\hline
\end{tabular}

$\operatorname{mid}\left(\mathbf{x}^{L}(t), \mathbf{x}^{U}(t), \mathbf{y}\right)$, respectively. Again, the quantities, $\alpha, \beta, \delta$ and $\gamma$ are defined as

$$
\begin{array}{ll}
\alpha_{8}=\min \left\{v_{9}^{L} v_{8}^{c}, v_{9}^{L} v_{8}^{C}\right\}, & \alpha_{9}=\min \left\{v_{8}^{L} v_{9}^{c}, v_{8}^{L} v_{9}^{C}\right\}, \\
\beta_{8}=\min \left\{v_{9}^{U} v_{8}^{c}, v_{9}^{U} v_{8}^{C}\right\}, & \beta_{9}=\min \left\{v_{8}^{U} v_{9}^{c}, v_{8}^{U} v_{9}^{C}\right\}, \\
\gamma_{8}=\max \left\{v_{9}^{L} v_{8}^{c}, v_{9}^{L} v_{8}^{C}\right\}, & \gamma_{9}=\max \left\{v_{8}^{U} v_{9}^{c}, v_{8}^{U} v_{9}^{C}\right\}, \\
\delta_{8}=\max \left\{v_{9}^{U} v_{8}^{c}, v_{9}^{U} v_{8}^{C}\right\}, & \delta_{9}=\max \left\{v_{8}^{L} v_{9}^{c}, v_{8}^{L} v_{9}^{C}\right\} .
\end{array}
$$

Now $u_{1}(t, \mathbf{p}, \mathbf{z}, \mathbf{y})$ and $o_{1}(t, \mathbf{p}, \mathbf{z}, \mathbf{y})$ evaluate to $v_{3}^{c}$ and $v_{3}^{C}$ in Table I, respectively, and $u_{2}(t, \mathbf{p}, \mathbf{z}, \mathbf{y})$ and $o_{2}(t, \mathbf{p}, \mathbf{z}, \mathbf{y})$ evaluate to $v_{10}^{c}$ and $v_{10}^{C}$ in Table II, respectively.

Given the functions $\mathbf{c}_{0}, \mathbf{C}_{0}, \mathbf{u}$ and $\mathbf{o}$ as described above, convex and concave relaxations for the parametric solution of (10) were generated by application of Theorem 4.1. The resulting relaxations are shown in Figure 2. Clearly, the minimum of the convex relaxation underestimates the global minimum of $x_{1}\left(t_{f}, \cdot\right)$. Figure 3 shows a second pair of convex and concave relaxations, plotted with the first, constructed in exactly the same way over the subinterval $P^{1}=[0.3,0.5]^{2}$ (the solution of (10) has been omitted for clarity). Clearly, the relaxations become much tighter when taken over a subinterval of the original parameter interval $P$.

\section{CONCLUSION}

Given a nonlinear system of ODEs (1), sufficient conditions have been established for a system of auxiliary differential equations of the form (2) to describe convex and concave relaxations of each state variable with respect to the ODE parameters, pointwise in the independent variable. Further, conditions have also been established under which such auxiliary systems lead to a consistent bounding operation in the sense of [7]. Thus, the state relaxations described here may be employed in spatial branch-and-bound global optimization procedures, and the resulting algorithms are finite $\varepsilon$-convergent. In a separate article [19], the authors presented a generalization of McCormick's relaxation technique which provides a computationally inexpensive and easily automatable method for generating auxiliary differential equations satisfying the sufficient conditions established in this article. Taken in conjunction with this work, the two provide a constructive procedure for automatically generating and evaluating convex and concave relaxations of the solutions of a very 


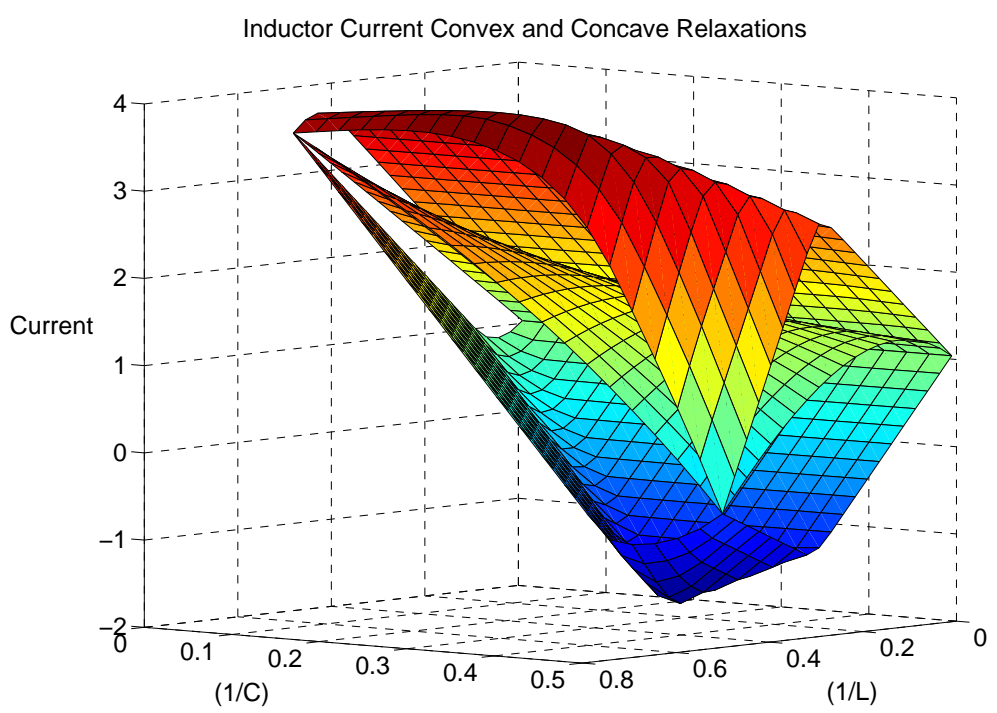

Figure 2. Nonlinear convex and concave relaxations of $x_{1}\left(t_{f}, \cdot\right)$, the solution of the ODEs (10), constructed over the interval $P=[0.01,0.5]^{2}$.

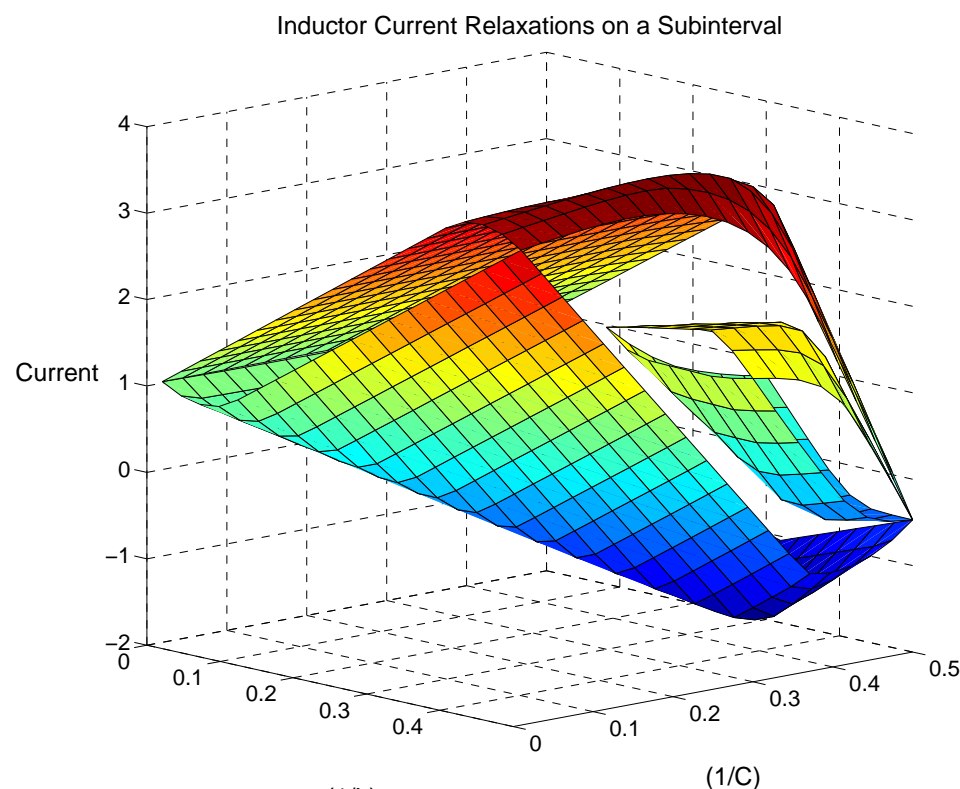

$(1 / L)$

$(1 / C)$

Figure 3. Nonlinear convex and concave relaxations of $x_{1}\left(t_{f}, \cdot\right)$, the solution of the ODEs (10), constructed over the interval $P=[0.01,0.5]^{2}$ and the subinterval $P^{1}=[0.3,0.5]^{2}$.

general class of nonlinear ODEs. Future work is under way to incorporate these relaxations into a deterministic global optimization algorithm for a general class of optimal control problems.

As discussed in detail in $\S 1$, this work considered ODEs influenced by a real parameter vector, as opposed to control functions, primarily due to the importance of such ODEs in algorithms for 
computational optimal control using control parametrization. However, owing to the central role played by convexity in the theory of optimal control and the calculus of variations [36, 1], there are potentially many other reasons, both theoretically and computationally, why it would be of interest to establish an analogous relaxation theory for ODEs influenced by $L^{p}$ control functions on $I$, taking values in the closed, bounded interval $P$. Indeed, the main convexity arguments used throughout this work are valid in more general vector spaces. This extension is currently under investigation by the authors.

\section{ACKNOWLEDGEMENT}

This paper is based on work funded by the National Science Foundation under grants CTS-0521962 and CBET-0933095.

\section{A. SUPPORTING MATERIAL}

This section contains some standard results in ODE theory [25], suitably modified for the purposes of this article. The statements of the results below differ from those in [25] (in fact from any presentation the authors are aware of) in the way that parameter dependence is incorporated and in the focus of Theorem A.1 on the existence of the successive approximations themselves, rather than on the existence and uniqueness result they are typically used to prove. For completeness, proofs are presented in full.

Theorem A.1

Consider a system of ODEs of the form (1), satisfying Assumption 3.1, and suppose that $D=\mathbb{R}^{n_{x}}$ and $\exists L \in \mathbb{R}_{+}$such that

$$
\|\mathbf{f}(t, \mathbf{p}, \mathbf{z})-\mathbf{f}(t, \mathbf{p}, \hat{\mathbf{z}})\|_{1} \leq L\|\mathbf{z}-\hat{\mathbf{z}}\|_{1}, \quad \forall(t, \mathbf{p}, \mathbf{z}, \hat{\mathbf{z}}) \in I \times P \times \mathbb{R}^{n_{x}} \times \mathbb{R}^{n_{x}}
$$

Given any continuous function $\mathrm{x}^{0}: I \times P \rightarrow \mathbb{R}^{n_{x}}$, the successive approximations defined recursively by

$$
\mathbf{x}^{k+1}(t, \mathbf{p})=\mathbf{x}_{0}(\mathbf{p})+\int_{t_{0}}^{t} \mathbf{f}\left(s, \mathbf{p}, \mathbf{x}^{k}(s, \mathbf{p})\right) d s
$$

exist as continuous functions on $I \times P$ and converge uniformly to a solution of (1) there. Furthermore, this solution is unique.

Proof

By hypothesis, $\mathbf{x}^{0}$ is defined and continuous on all of $I \times P$. Supposing this is true of $\mathbf{x}^{k}$ and noting that $\mathbf{x}^{k}(t, \mathbf{p})$ is trivially an element of $D$ for all $(t, \mathbf{p}) \in I \times P,(12)$ defines $\mathbf{x}^{k+1}$ on all of $I \times P$ and continuity follows from the continuity of $\mathbf{x}_{0}$ and $\mathbf{f}$. Thus, induction shows that each $\mathbf{x}^{k}$ is defined and continuous on all of $I \times P$.

Now define

$$
\gamma \equiv \max _{(t, \mathbf{p}) \in I \times P}\left\|\mathbf{f}\left(t, \mathbf{p}, \mathbf{x}^{1}(t, \mathbf{p})\right)-\mathbf{f}\left(t, \mathbf{p}, \mathbf{x}^{0}(t, \mathbf{p})\right)\right\|_{1}
$$

It will be shown that

$$
\left\|\mathbf{x}^{k+1}(t, \mathbf{p})-\mathbf{x}^{k}(t, \mathbf{p})\right\|_{1} \leq \frac{\gamma L^{k}\left(t-t_{0}\right)^{k}}{L k !}
$$


for all $(t, \mathbf{p}) \in I \times P$ and every $k \in \mathbb{N}$. For $k=1$, (12) directly gives

$$
\left\|\mathbf{x}^{2}(t, \mathbf{p})-\mathbf{x}^{1}(t, \mathbf{p})\right\|_{1} \leq \int_{t_{0}}^{t}\left\|\mathbf{f}\left(s, \mathbf{p}, \mathbf{x}^{1}(s, \mathbf{p})\right)-\mathbf{f}\left(s, \mathbf{p}, \mathbf{x}^{0}(s, \mathbf{p})\right)\right\|_{1} d s \leq \gamma\left(t-t_{0}\right),
$$

for all $(t, \mathbf{p}) \in I \times P$. Supposing that (13) holds for some arbitrary $k$, it must also hold for $k+1$ since

$$
\begin{aligned}
\left\|\mathbf{x}^{k+2}(t, \mathbf{p})-\mathbf{x}^{k+1}(t, \mathbf{p})\right\|_{1} & \leq \int_{t_{0}}^{t}\left\|\mathbf{f}\left(s, \mathbf{p}, \mathbf{x}^{k+1}(s, \mathbf{p})\right)-\mathbf{f}\left(s, \mathbf{p}, \mathbf{x}^{k}(s, \mathbf{p})\right)\right\|_{1} d s \\
& \leq L \int_{t_{0}}^{t}\left\|\mathbf{x}^{k+1}(s, \mathbf{p})-\mathbf{x}^{k}(s, \mathbf{p})\right\|_{1} d s \\
& \leq \frac{\gamma L^{k+1}}{L k !} \int_{t_{0}}^{t}\left(s-t_{0}\right)^{k} d s \\
& \leq \frac{\gamma L^{k+1}\left(t-t_{0}\right)^{k+1}}{L(k+1) !}
\end{aligned}
$$

for all $(t, \mathbf{p}) \in I \times P$. Thus, induction proves (13). Now, for any $n, m \in \mathbb{N}$ with $m>n$, Equation (13) and the triangle inequality give

$$
\begin{aligned}
\left\|\mathbf{x}^{n}(t, \mathbf{p})-\mathbf{x}^{m}(t, \mathbf{p})\right\|_{1} & \leq\left\|\mathbf{x}^{n+1}(t, \mathbf{p})-\mathbf{x}^{n}(t, \mathbf{p})\right\|_{1}+\ldots+\left\|\mathbf{x}^{m}(t, \mathbf{p})-\mathbf{x}^{m-1}(t, \mathbf{p})\right\|_{1} \\
& \leq \frac{\gamma L^{n}\left(t_{f}-t_{0}\right)^{n}}{L n !}+\ldots+\frac{\gamma L^{m-1}\left(t_{f}-t_{0}\right)^{m-1}}{L(m-1) !} \\
& \leq \sum_{k=n}^{\infty} \frac{\gamma L^{k}\left(t_{f}-t_{0}\right)^{k}}{L k !}
\end{aligned}
$$

for all $(t, \mathbf{p}) \in I \times P$. But

$$
\sum_{k=0}^{\infty} \frac{\gamma L^{k}\left(t_{f}-t_{0}\right)^{k}}{L k !}=\frac{\gamma}{L} e^{L\left(t_{f}-t_{0}\right)}<\infty
$$

and hence $\lim _{n \rightarrow \infty} \sum_{k=n}^{\infty} \frac{\gamma L^{k}\left(t_{f}-t_{0}\right)^{k}}{L k !}=0$, which implies that the sequence $\left\{\mathbf{x}^{k}\right\}$ is uniformly Cauchy on $I \times P$, and hence converges uniformly to a continuous limit function there.

Next it is shown that this limit function, denoted $\mathbf{x}$, is a solution of (1) on $I \times P$. From the Lipschitz condition on $\mathbf{f}$,

$$
\left\|\int_{t_{0}}^{t} \mathbf{f}\left(s, \mathbf{p}, \mathbf{x}^{k}(s, \mathbf{p})\right) d s-\int_{t_{0}}^{t} \mathbf{f}(s, \mathbf{p}, \mathbf{x}(s, \mathbf{p})) d s\right\|_{1} \leq L \int_{t_{0}}^{t}\left\|\mathbf{x}^{k}(s, \mathbf{p})-\mathbf{x}(s, \mathbf{p})\right\|_{1} d s
$$

for all $(t, \mathbf{p}) \in I \times P$, so that the uniform convergence of $\left\{\mathbf{x}^{k}\right\}$ to $\mathbf{x}$ on $I \times P$ implies that $\lim _{k \rightarrow \infty} \int_{t_{0}}^{t} \mathbf{f}\left(s, \mathbf{p}, \mathbf{x}^{k}(s, \mathbf{p})\right) d s=\int_{t_{0}}^{t} \mathbf{f}(s, \mathbf{p}, \mathbf{x}(s, \mathbf{p})) d s$, for all $(t, \mathbf{p}) \in I \times P$. Then, taking limits on both sides of (12) gives

$$
\mathbf{x}(t, \mathbf{p})=\mathbf{x}_{0}(\mathbf{p})+\int_{t_{0}}^{t} \mathbf{f}(s, \mathbf{p}, \mathbf{x}(s, \mathbf{p})) d s, \quad \forall(t, \mathbf{p}) \in I \times P
$$

which, by the fundamental theorem of calculus and continuity of the integrand, implies that $\mathrm{x}$ is a solution of (1). Uniqueness of $\mathbf{x}$ now follows (for each fixed $\mathbf{p} \in P$ ), by a standard application of Gronwall's inequality (Theorem 1.1, Ch. III, [37]).

The notation in the following Lemma is from $\S 4.1$. 
Lemma A.1

If $\left\{\mathbf{u}^{\ell}\right\} \rightarrow \mathbf{u}^{*}$ and $\left\{\mathbf{o}^{\ell}\right\} \rightarrow \mathbf{o}^{*}$ uniformly on $I \times P \times \mathbb{R}^{n_{x}} \times \mathbb{R}^{n_{x}}$ and $\left\{\mathbf{c}_{0}^{\ell}\right\} \rightarrow \mathbf{c}_{0}^{*}$ and $\left\{\mathbf{C}_{0}^{\ell}\right\} \rightarrow \mathbf{C}_{0}^{*}$ uniformly on $P$, then $\left\{\mathbf{c}^{\ell}\right\} \rightarrow \mathbf{c}^{*}$ and $\left\{\mathbf{C}^{\ell}\right\} \rightarrow \mathbf{C}^{*}$ uniformly on $I \times P$.

Proof

From the uniform convergence of $\mathbf{c}^{\ell}, \mathbf{C}^{\ell}, \mathbf{u}^{\ell}$ and $\mathbf{o}^{\ell}$, given any $\varepsilon, \delta>0$, there exists a positive integer $N$ such that, for $\ell \geq N$,

$$
\left\|\mathbf{c}_{0}^{\ell}(\mathbf{p})-\mathbf{c}_{0}^{*}(\mathbf{p})\right\|_{1}+\left\|\mathbf{C}_{0}^{\ell}(\mathbf{p})-\mathbf{C}_{0}^{*}(\mathbf{p})\right\|_{1} \leq \delta
$$

and

$$
\begin{aligned}
\left\|\mathbf{u}^{\ell}\left(t, \mathbf{p}, \mathbf{c}^{\ell}(t, \mathbf{p}), \mathbf{C}^{\ell}(t, \mathbf{p})\right)-\mathbf{u}^{*}\left(t, \mathbf{p}, \mathbf{c}^{\ell}(t, \mathbf{p}), \mathbf{C}^{\ell}(t, \mathbf{p})\right)\right\|_{1}+ \\
\left\|\mathbf{o}^{\ell}\left(t, \mathbf{p}, \mathbf{c}^{\ell}(t, \mathbf{p}), \mathbf{C}^{\ell}(t, \mathbf{p})\right)-\mathbf{o}^{*}\left(t, \mathbf{p}, \mathbf{c}^{\ell}(t, \mathbf{p}), \mathbf{C}^{\ell}(t, \mathbf{p})\right)\right\|_{1} \leq \varepsilon,
\end{aligned}
$$

for all $(t, \mathbf{p}) \in I \times P$. Integrating both sides of the second inequality from $t_{0}$ to $t$ gives

$$
\begin{aligned}
& \left\|\mathbf{c}^{\ell}(t, \mathbf{p})-\mathbf{c}_{0}^{\ell}(\mathbf{p})-\int_{t_{0}}^{t} \mathbf{u}^{*}\left(s, \mathbf{p}, \mathbf{c}^{\ell}(s, \mathbf{p}), \mathbf{C}^{\ell}(s, \mathbf{p})\right) d s\right\|_{1}+ \\
& \left\|\mathbf{C}^{\ell}(t, \mathbf{p})-\mathbf{C}_{0}^{\ell}(\mathbf{p})-\int_{t_{0}}^{t} \mathbf{o}^{*}\left(s, \mathbf{p}, \mathbf{c}^{\ell}(s, \mathbf{p}), \mathbf{C}^{\ell}(s, \mathbf{p})\right) d s\right\|_{1} \leq \varepsilon\left(t-t_{0}\right) .
\end{aligned}
$$

Noting that

$$
\begin{aligned}
& \left\|\mathbf{c}^{*}(t, \mathbf{p})-\mathbf{c}_{0}^{*}(\mathbf{p})-\int_{t_{0}}^{t} \mathbf{u}^{*}\left(s, \mathbf{p}, \mathbf{c}^{*}(s, \mathbf{p}), \mathbf{C}^{*}(s, \mathbf{p})\right) d s\right\|_{1}+ \\
& \left\|\mathbf{C}^{*}(t, \mathbf{p})-\mathbf{C}_{0}^{*}(\mathbf{p})-\int_{t_{0}}^{t} \mathbf{o}^{*}\left(s, \mathbf{p}, \mathbf{c}^{*}(s, \mathbf{p}), \mathbf{C}^{*}(s, \mathbf{p})\right) d s\right\|_{1}=0,
\end{aligned}
$$

for all $(t, \mathbf{p}) \in I \times P$, the identity $\|\boldsymbol{\alpha}-\boldsymbol{\beta}\|_{1}+\|\boldsymbol{\gamma}-\boldsymbol{\delta}\|_{1} \leq\|\boldsymbol{\alpha}\|_{1}+\|\boldsymbol{\beta}\|_{1}+\|\boldsymbol{\gamma}\|_{1}+\|\boldsymbol{\delta}\|_{1}$ gives

$$
\begin{array}{r}
\|\left(\mathbf{c}^{\ell}(t, \mathbf{p})-\mathbf{c}^{*}(t, \mathbf{p})\right)-\left(\mathbf{c}_{0}^{\ell}(\mathbf{p})-\mathbf{c}_{0}^{*}(\mathbf{p})\right)- \\
\int_{t_{0}}^{t}\left[\mathbf{u}^{*}\left(s, \mathbf{p}, \mathbf{c}^{\ell}(s, \mathbf{p}), \mathbf{C}^{\ell}(s, \mathbf{p})\right)-\mathbf{u}^{*}\left(s, \mathbf{p}, \mathbf{c}^{*}(s, \mathbf{p}), \mathbf{C}^{*}(s, \mathbf{p})\right)\right] d s \|_{1}+ \\
\|\left(\mathbf{C}^{\ell}(t, \mathbf{p})-\mathbf{C}^{*}(t, \mathbf{p})\right)-\left(\mathbf{C}_{0}^{\ell}(\mathbf{p})-\mathbf{C}_{0}^{*}(\mathbf{p})\right)- \\
\int_{t_{0}}^{t}\left[\mathbf{o}^{*}\left(s, \mathbf{p}, \mathbf{c}^{\ell}(s, \mathbf{p}), \mathbf{C}^{\ell}(s, \mathbf{p})\right)-\mathbf{o}^{*}\left(s, \mathbf{p}, \mathbf{c}^{*}(s, \mathbf{p}), \mathbf{C}^{*}(s, \mathbf{p})\right)\right] d s \|_{1} \\
\leq \varepsilon\left(t-t_{0}\right) .
\end{array}
$$

Let $r(t, \mathbf{p}) \equiv\left\|\mathbf{c}^{\ell}(t, \mathbf{p})-\mathbf{c}^{*}(t, \mathbf{p})\right\|_{1}$ and $q(t, \mathbf{p}) \equiv\left\|\mathbf{C}^{\ell}(t, \mathbf{p})-\mathbf{C}^{*}(t, \mathbf{p})\right\|_{1}$. Substituting these definitions into the previous inequality and noting that $\|\boldsymbol{\alpha}\|_{1}-\|\boldsymbol{\beta}\|_{1} \leq\|\boldsymbol{\alpha}-\boldsymbol{\beta}\|_{1}$,

$$
\begin{aligned}
r(t, \mathbf{p})+q(t, \mathbf{p}) \leq \delta & +\int_{t_{0}}^{t} \| \mathbf{u}^{*}\left(s, \mathbf{p}, \mathbf{c}^{\ell}(s, \mathbf{p}), \mathbf{C}^{\ell}(s, \mathbf{p})\right) \\
& -\mathbf{u}^{*}\left(s, \mathbf{p}, \mathbf{c}^{*}(s, \mathbf{p}), \mathbf{C}^{*}(s, \mathbf{p})\right) \|_{1} d s \\
+ & \int_{t_{0}}^{t} \| \mathbf{o}^{*}\left(s, \mathbf{p}, \mathbf{c}^{\ell}(s, \mathbf{p}), \mathbf{C}^{\ell}(s, \mathbf{p})\right) \\
& -\mathbf{o}^{*}\left(s, \mathbf{p}, \mathbf{c}^{*}(s, \mathbf{p}), \mathbf{C}^{*}(s, \mathbf{p})\right) \|_{1} d s+\varepsilon\left(t-t_{0}\right),
\end{aligned}
$$


and because $\mathbf{u}^{*}(t, \mathbf{p}, \cdot, \cdot)$ and $\mathbf{o}^{*}(t, \mathbf{p}, \cdot, \cdot)$ are Lipschitz on $\mathbb{R}^{2 n_{x}}$ uniformly on $I \times P$,

$$
r(t, \mathbf{p})+q(t, \mathbf{p}) \leq\left(\delta+\varepsilon\left(t_{f}-t_{0}\right)\right)+L_{\mathbf{u o}} \int_{t_{0}}^{t}(r(s, \mathbf{p})+q(s, \mathbf{p})) d s,
$$

for all $(t, \mathbf{p}) \in I \times P$. Now Gronwall's inequality (Theorem 1.1, Ch. III, [37]) gives

$$
r(t, \mathbf{p})+q(t, \mathbf{p}) \leq\left(\delta+\varepsilon\left(t_{f}-t_{0}\right)\right) e^{L_{\mathbf{u o}}\left(t-t_{0}\right)}, \quad \forall(t, \mathbf{p}) \in I \times P .
$$

Substituting $t=t_{f}$ in the right-hand side gives a uniform upper bound on $I \times P$, and since $\varepsilon$ and $\delta$ can be made arbitrarily small as $\ell \rightarrow \infty, r(t, \mathbf{p}) \rightarrow 0$ and $q(t, \mathbf{p}) \rightarrow 0$ uniformly on $I \times P$. Therefore, $\left\{\mathbf{c}^{\ell}\right\} \rightarrow \mathbf{c}^{*}$ and $\left\{\mathbf{C}^{\ell}\right\} \rightarrow \mathbf{C}^{*}$ uniformly on $I \times P$.

\section{REFERENCES}

1. Troutman JL. Variational Calculus and Optimal Control: Optimzation with Elementary Convexity. second edn., Springer-Verlag: New York, 1996.

2. Singer AB, Barton PI. Global optimization with nonlinear ordinary differential equations. J. Glob. Optim. 2006; 34:159-190.

3. Papamichail I, Adjiman CS. A rigorous global optimization algorithm for problems with ordinary differential equations. J. Glob. Optim. 2002; 24(1):1-33.

4. KTeo, Goh G, Wong K. A Unified Computational Approach to Optimal Control Problems. John Wiley and Sons, Inc.: New York, 1991.

5. Goh CJ, Teo KL. Control parametrization: a unified approach to optimal control problems with general constraints. Automatica 1988; 24(1):3-18.

6. Lee HWJ, Teo KL, Rehbock V, Jennings LS. Control parametrization enhancing technique for time optimal control problems. Dynam. Systems Appl. 1997; 6:243-261.

7. Horst R, Tuy H. Global Optimization: Deterministic Approaches. third edn., Springer: New York, 1996.

8. Tawarmalani M, Sahinidis NV. Convexification and Global Optimization in Continuous and Mixed-Integer Nonlinear Programming. Kluwer Academic Publishers, 2002.

9. Kesavan P, Allgor RJ, Gatzke EP, Barton PI. Outer approximation algorithms for separable nonconvex mixedinteger nonlinear programs. Math. Program. December 2004; 100:517-535.

10. Chachuat B, Singer AB, Barton PI. Global mixed-integer dynamic optimization. AIChE Journal 2005; 51(8):22352253.

11. McCormick GP. Computability of global solutions to factorable nonconvex programs: Part I - convex underestimating problems. Math. Program. 1976; 10:147-175.

12. Androulakis IP, Maranas CD, Floudas CA. $\alpha$ BB: A global optimization method for general constrained nonconvex problems. J. Glob. Optim. 1995; 7:337-363.

13. Esposito WR, Floudas CA. Deterministic global optimization in nonlinear optimal control problems. J. Glob. Optim. 2000; 17:97-126.

14. Singer AB, Barton PI. Bounding the solutions of parameter dependent nonlinear ordinary differential equations. SIAM J. Sci. Comput. 2006; 27:2167-2182.

15. Mitsos A, Chachuat B, Barton PI. McCormick-Based Relaxations of Algorithms. SIAM J. on Optim. 2009; 20(2):573-601.

16. Sahlodin AM, Chachuat B. Discretize-then-relax approach for convex/concave relaxations of the solutions of parametric ODEs. Applied Numerical Mathematics 2011; 61:803-820.

17. Sahlodin AM, Chachuat B. Convex/concave relaxations of parametric odes using taylor models. Comp. and Chem. Eng. 2011; 35:844-857.

18. Lin Y, Stadtherr MA. Deterministic global optimization of nonlinear dynamic systems. AIChE Journal 2007; 53(4):866-875.

19. Scott JK, Stuber MD, Barton PI. Generalized McCormick relaxations. J. Glob. Optim., In Press 2011; doi: 10.1007/s10898-011-9664-7. Doi: 10.1007/s10898-011-9664-7.

20. Azhmyakov V, Raisch J. Convex control systems and convex optimal control. IEEE Trans. Automat. Contr. 2008; 53(4):993-998. 
21. Seierstad A, Sydstaeter K. Sufficient conditions in optimal control theory. International Economic Review 1977; 18(2):367-391.

22. Reissig G. Convexity of reachable sets of nonlinear ordinary differential equations. Automation and Remote Control 2007; 68(9):64-78.

23. Warga J. Optimal Control of Differential and Functional Equations. Academic Press, Inc.: New York, 1972.

24. Fonseca I, Leoni G. Modern Methods in the Calculus of Variations: $L^{p}$ Spaces. Springer Monographs in Mathematics, Springer: New York, 2007.

25. Coddington EA, Levinson N. Theory of Ordinary Differential Equations. McGraw-Hill: New York, 1955.

26. Khalil KH. Nonlinear Systems. Third edn., Prentice Hall: Upper Saddle River, NJ, 2002.

27. Moore RE. Methods and Applications of Interval Analysis. SIAM: Philadelphia, PA, 1979.

28. Chachuat B. MC++: A numeric library for McCormick relaxation of factorable functions. Documentation and Source Code available at: http://www3.imperial.ac.uk/people/b.chachuat/research 2011; .

29. Cohen SD, Hindmarsh AC. CVODE, A Stiff/Nonstiff ODE Solver in C. Computers in Physics 1996; 10(2):138143.

30. Harrison GW. Dynamic models with uncertain parameters. Proc. of the First International Conference on Mathematical Modeling, vol. 1, Avula X (ed.), 1977; 295-304.

31. Singer AB, Barton PI. Global solution of optimization problems with parameter-embedded linear dynamic systems. J. Optim. Theory Appl. 2004; 121:613-646.

32. Scott JK, Barton PI. Tight, efficient bounds on the solutions of chemical kinetics models. Computers and Chemical Engineering 2010; 34:717-731.

33. Lin Y, Stadtherr MA. Validated Solutions of initial value problems for parametric ODEs. Applied Numerical Mathematics 2007; 57:1145-1162.

34. Nedialkov NS, Jackson KR, Corliss GF. Validated solutions of initial value problems for ordinary differential equations. Appl. Math. Comput. 1999; 105:21-68.

35. Liberti L, Pantelides CC. Convex envelopes of monomials of odd degree. J. Glob. Optim. 2003; 25:157-168.

36. Berkovitz LD. Optimal Control Theory. Springer: New York, 1974.

37. Hartman P. Ordinary differential equations. second edn., SIAM: Philidelphia, PA, 2002. 